\title{
SPIDER RECORDS FROM FOUR WILDLAND PARKS IN NORTHEASTERN ALBERTA
}

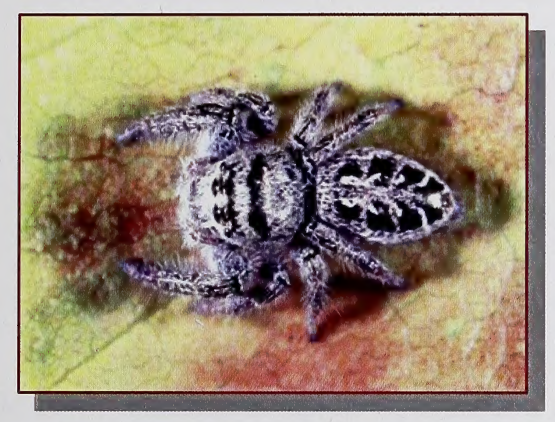

Eris militaris (female) Photo by D. Buckle

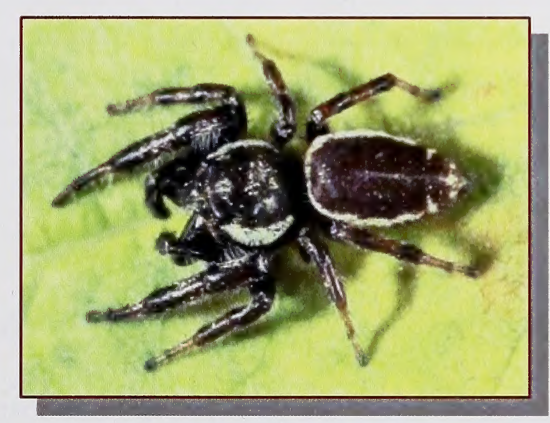

Eris militaris (male) Photo by D. Buckle

Prepared by
Wayne Nordstrom $^{1}$ and Don Buckle

May 2002

${ }^{1}$ Alberta Natural Heritage Information Centre

Parks and Protected Areas Division

Alberta Community Development

${ }^{2} 620$ Albert Avenue

Saskatoon, SK S7N 1G7 


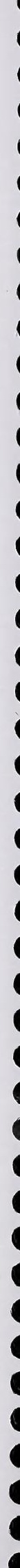




\section{Table of Contents}

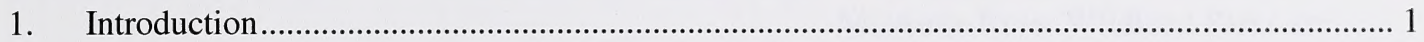

2. Methods...

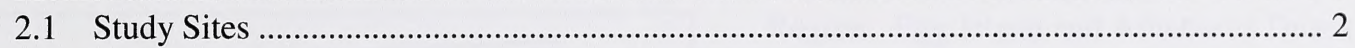

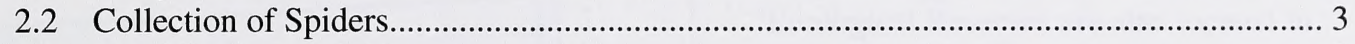

2.3 Identification of Spiders......................................................................................... 3

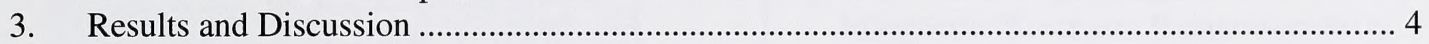

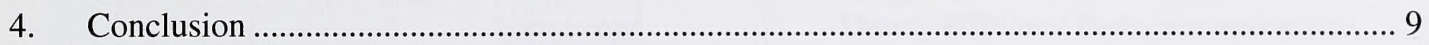

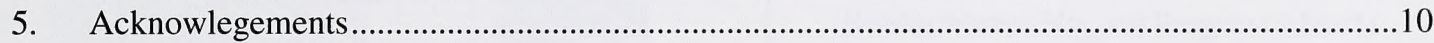

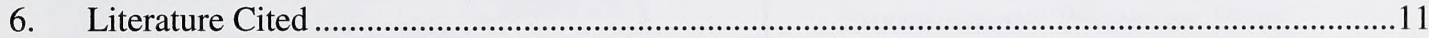

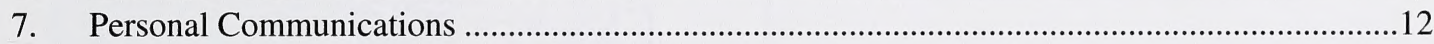

\section{List of Tables}

Table 1. Number of spider species and specimens in each family that were collected from four wildland parks in northeastern Alberta .......................................................................... 4

Table 2. Adult male, adult female and immature specimens in each of the 15 spider families represented in the four wildland parks of northeastern Alberta........................................ 6

\section{List of Figures}

Figure 1. Wildland parks in northeastern Alberta ................................................................. 1

Figure 2. Spiders collected within four wildland parks in northeastern Alberta............................... 5

Figure 3. Spider species for which four or more specimens were collected ..................................... 6

Figure 4. The number of adult specimens collected per species ................................................... 7

Figure 5. The number of adult specimens collected within each of the wildland parks ................. 7

\section{Appendices}

Appendix 1. Annotated notes for the spiders that were collected from four wildland parks in northeastern Alberta during 2000 and 2001

Appendix 2. Spiders collected from four wildland parks in northeastern Alberta during 2000 and 2001 



\section{Introduction}

Several wildland parks and two ecological reserves have recently been established in the northeast corner of Alberta (Figure 1). Colin-Cornwall Lakes Fidler-Greywillow, La Butte Creek and

Maybelle River Wildland Parks are located in the Canadian Shield Natural

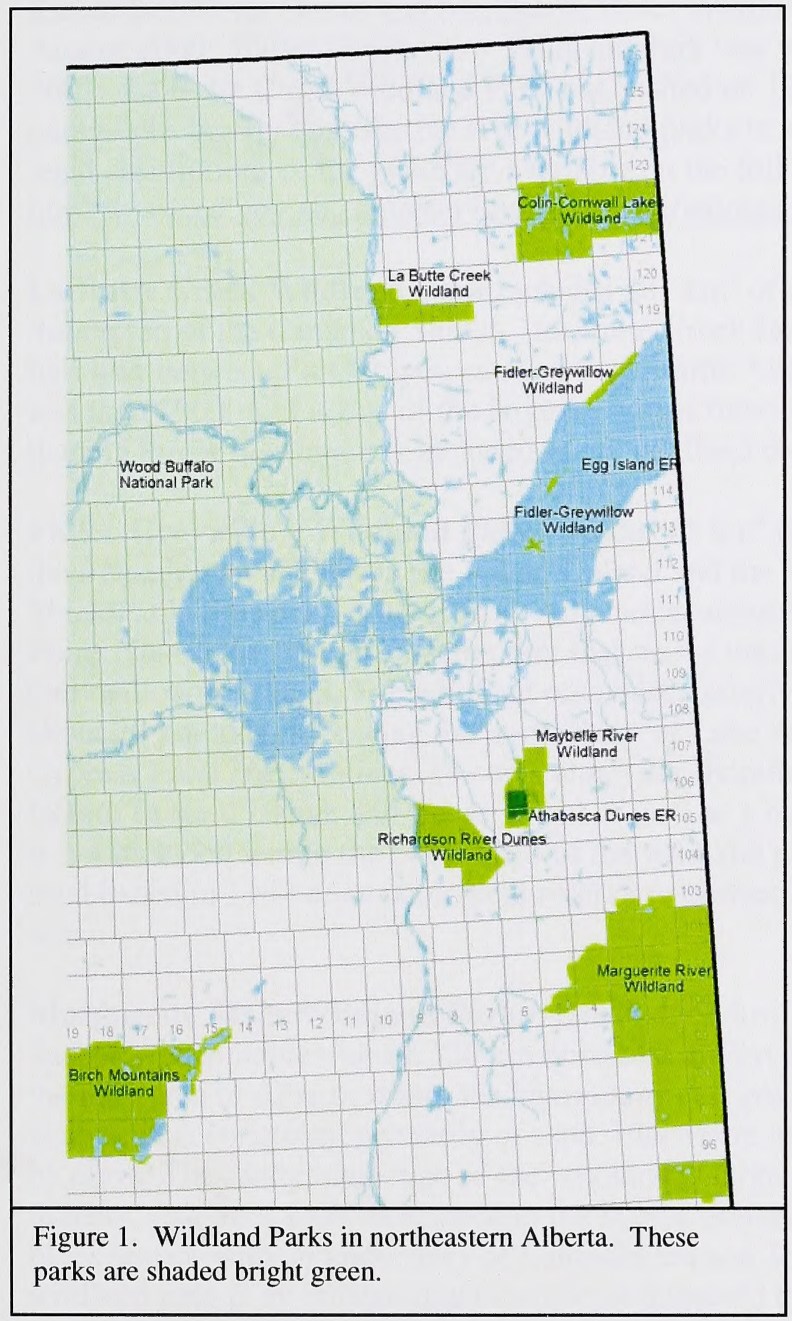
Region. Egg Island and Athabasca Dunes Ecological Reserves are also located in that Natural Region. Birch Mountains, Marguerite River and Richardson River Dunes Wildland Parks are within the Boreal Forest Natural Region (Alberta Environmental Protection 1998).

These wildland parks contain numerous and important examples of Alberta's biodiversity. Much of it is poorly understood or unknown, particularly the invertebrate fauna. The spider fauna is no exception. As Aitchison and Sutherland (2000) state, "...information on the composition and functioning of the boreal forest arachnid community [in Canada] remains sketchy".

The spider specimens that were collected during this survey are the first for these wildland parks of northeastern Alberta. (R. Holmberg, R. Leech, pers. comm.).

In Canada there are approximately 1400 known spider species (Bennett 1999, Aitchison and Sutherland 2000). The Biological Survey of Canada estimates that there are at most 1,500 spider species in the country (Bennett 1999). Within Alberta, there are 526 known spider species in 22 families, with another 100 or so species to be collected or described (Buckle and Holmberg 2001).

During his work at the George Lake Field Station west of Busby, Alberta and at other sites throughout north-central Alberta, Buddle (2000) found a total of 160 species of spiders. This represents approximately $30 \%(160 / 526)$ of the total number of known species for the province. 


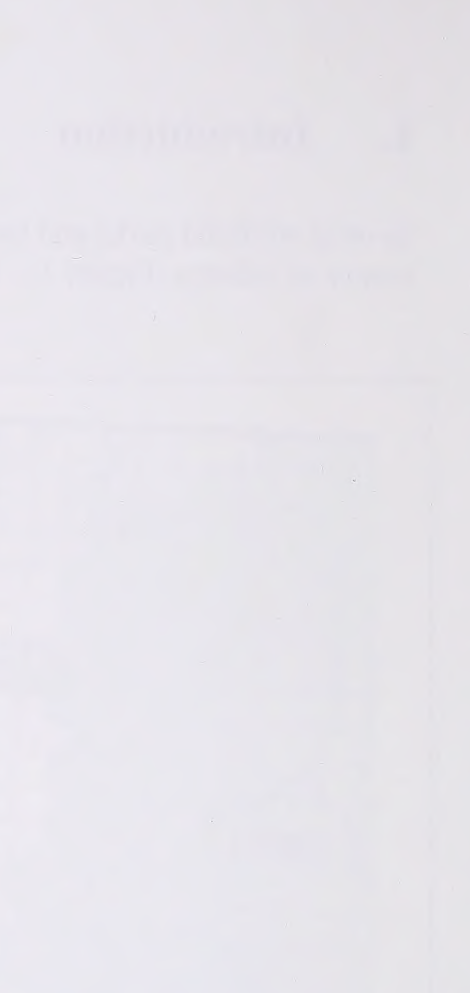




\section{Methods}

\subsection{Study Sites:}

Field surveys for five of the seven wildland parks shown in Figure 1 were conducted by staff of the Parks and Protected Areas Division during the summers of 2000 and 2001 . Maybelle River, Richardson River Dunes and Marguerite River Wildland Parks were visited on 12-24 June and 22-25 August 2000. Fidler-Greywillow Wildland Park was visited on 6-8 June, 10-12 June and 17-26 July 2001. La Butte Creek Wildland Park was visited on 13-16 June and 5-15 July 2001. The following paragraphs briefly describe the five wildland parks in which field surveys were conducted. Maps and legal descriptions of the parks are available on the following URL:

http://www.cd.gov.ab.ca/preserving/parks/lrm/wildland/wildpark.asp.

La Butte Creek Wildland Park includes $181 \mathrm{~km}^{2}$ of uplands and wetlands in the Kazan Upland Subregion of the Canadian Shield. La Butte Creek flows into the Slave River from the east about half way between Fort Chipewyan and Fort Smith. Although the entire length of La Butte Creek is less than $100 \mathrm{~km}$, it is one of the largest streams flowing from the Kazan Upland in Alberta. More than $35 \mathrm{~km}$ of the stream is included in the wildland park.

Fidler-Greywillow Wildland Park includes $65 \mathrm{~km}^{2}$ of the shore and islands in Lake Athabasca at the transition area between the Kazan Upland and the Athabasca Plain Subregion of the Canadian Shield. On the north shore of Lake Athabasca, about $80 \mathrm{~km}$ northeast of Fort Chipewyan, is Fidler Point. This is the first prominent point that marks the southwestern edge of the main part of FidlerGreywillow Wildland. The wildland runs northeasterly from this point in a strip about $3 \mathrm{~km}$ wide and about $20 \mathrm{~km}$ long, following the north shore of Lake Athabasca. Fidler Point, Whitesand Point and Cypress Point are prominent features along the shoreline. The wildland also includes Burntwood Island, Bustard Island, and Lucas Island as well as a number of smaller islands. Egg Island, however, is not included within the boundaries of the wildland park. This small island of 0.36 ha was established in 1992 as an ecological reserve to protect Alberta's largest nesting colony of Caspian terns.

Marguerite River Wildland Park includes $1963 \mathrm{~km}^{2}$ of unique landscape located along the Saskatchewan border, about $130 \mathrm{~km}$ northeast of Fort McMurray. The landscape encompasses part of the only area in Alberta where the hummocky and ground moraine deposited by continental glaciation is composed primarily of sand. Elsewhere in Alberta these types of moraines are dominated by clays. The sandy landscape of the wildland is largely forested by jack pine with a ground cover of lichens. Peatlands have developed in the poorly drained depressions. These support forests of stunted black spruce with an understory of Labrador tea and Sphagnum mosses. Also included in the wildland park is an outstanding example of dissected kame, an extremely rugged landscape with local relief in excess of 200 meters. Other significant landform features of the park include braided streams, drumlins, glacial flutings, eskers, and "crag and tail" (i.e., granite crags with glacially deposited gravel tails on the lee side).

Maybelle River Wildland Park includes $153 \mathrm{~km}^{2}$ of sand plains, rugged kames and small kettle lakes in an area about $150 \mathrm{~km}$ north of Fort McMurray. Parts of the sand-dominated landscape have been reworked by winds to form impressive sand dunes. Alberta's largest active dune field occurs within the wildland park. This dune field measures about $8 \mathrm{~km}$ from north to south and is about 1.5 $\mathrm{km}$ wide. The meandering Maybelle River is the largest waterway that flows through the park. The 
well-drained sandy uplands are forested by jack pine with a ground cover of lichens. Peatlands have formed in the wet depressions, some adjacent to or surrounding the kettle lakes.

Richardson River Dunes Wildland Park is $320 \mathrm{~km}^{2}$ in size and includes part of the largest sand dune complex in Canada. The wildland park is located east of the Athabasca River about $40 \mathrm{~km}$ south of Lake Athabasca or $130 \mathrm{~km}$ north of Fort McMurray. Within the park are impressive parallel sand ridges, trending northwest to southeast. These are forested by jack pine with a ground cover of lichens. Small blowouts of unvegetated sand occur on the tops of some of the dune ridges. Extensive areas within the park have been burned and are vegetated by dense stands of young jack pine with few understory species. Burned areas have considerable deadfall. Small sedge meadows have developed in the troughs between the ridges.

\subsection{Collection of Spiders:}

Spiders were randomly collected from La Butte Creek, Fidler-Greywillow, Richardson River Dunes and Marguerite River Wildland Parks during the course of inventory work that focused on other taxa (i.e., birds, mammals, butterflies, moths and plants). Collection efforts relative to the spiders, therefore, were not standardized. Nevertheless, specimens were collected in these parks from a variety of habitats, including riparian areas, meadows, forests, lakeshores and disturbed sites. Spiders were not collected from Maybelle River Wildland Park.

Some spiders were obtained by sweep-netting low bushes, branches, grasses and sedges with an insect net while others were collected by hand. All specimens were preserved in $95 \%$ ethanol.

In addition to sweeps and hand collecting, pan traps were used for collecting arthropods (Finnamore et al. 2001). The pan traps were shallow relatively large plastic bowls (273 mm diameter x $79 \mathrm{~mm}$ depth) and were yellow in color (safety yellow in North America). These traps were placed randomly in depressions dug in beach / shoreline habitats and as well as in upland forested areas. Care was taken to minimize disturbance to the adjacent microhabitats. The rim of the pan trap was set level with the soil or litter surface. Its outside surrounding edge was filled with soil and detritus from the excavation and carefully worked flush with the rim. This provided a surface for arthropods to travel unimpeded up to the rim and into the trap. Soil and other detritus were removed from the trap and water was added. A few drops of detergent were added to the water as a surfactant. Flagging tape was used to mark the trap's location. Traps were serviced every two to four days, at which time arthropods were removed by seiving through fine-mesh cloth, rinsed in water and placed in labelled vials containing $95 \%$ ethanol.

\subsection{Identification of Spiders:}

Spider specimens were identified by the junior author and will be deposited in the E.H. Strickland Entomological Museum in the Earth Sciences Building at the University of Alberta in Edmonton. 


\section{Results and Discussion}

There were 80 spider species collected during this study from 15 spider families (Table 1). This is $15 \%(80 / 526)$ of the spider species known to occur in Alberta. Another 10 species may have been present but identification was impossible because only immatures were collected (e.g., two dictynids and 4 linyphiids). In their work in northern Manitoba, Aitchison and Sutherland (2000) collected only 49 spider species from 13 families, which is considerably less than what was obtained in the present study.

Table 1. Number of spider species and specimens in each family that were collected from four wildland parks in northeastern Alberta. Families are listed in descending order of abundance of specimens. All of the specimens were adults except for the four immatures of one species that were collected in the Family Clubionidae.

\begin{tabular}{|c|c|c|c|c|}
\hline \multirow[b]{2}{*}{ Family } & \multicolumn{2}{|c|}{ Species } & \multicolumn{2}{|c|}{ Specimens } \\
\hline & Number & Percent & Number & Percent \\
\hline Lycosidae & 14 & 17.5 & 56 & 23.6 \\
\hline Linyphiidae & 20 & 25.0 & 38 & 16.0 \\
\hline Tetragnathidae & 5 & 6.3 & 27 & 11.4 \\
\hline Araneidae & 10 & 12.5 & 22 & 9.3 \\
\hline Hahniidae & 2 & 2.5 & 18 & 7.6 \\
\hline Salticidae & 4 & 5.0 & 17 & 7.2 \\
\hline Gnaphosidae & 5 & 6.3 & 16 & 6.7 \\
\hline Agelenidae & 1 & 1.2 & 14 & 5.9 \\
\hline Dictynidae & 5 & 6.3 & 10 & 4.2 \\
\hline Philodromidae & 6 & 7.5 & 7 & 2.9 \\
\hline Clubionidae & 1 & 1.2 & 4 (immatures) & 1.7 \\
\hline Theridiidae & 2 & 2.5 & 3 & 1.3 \\
\hline Thomisidae & 3 & 3.8 & 3 & 1.3 \\
\hline Liocranidae & 1 & 1.2 & 1 & 0.4 \\
\hline Titanoecidae & 1 & 1.2 & 1 & 0.4 \\
\hline TOTAL & 80 & 100 & 237 & 100 \\
\hline
\end{tabular}

The three most commonly collected families in this study were the Lycosidae, Linyphiidae and Tetragnathidae (Table 1, Figure 2). The least commonly collected families were the Theridiidae, Thomisidae, Liocranidae and Titanoecidae. Aitchison and Sutherland (2000) found that the Lycosidae was the most commonly collected family in northern Manitoba followed by the Gnaphosidae and Agelenidae. Their least collected families included the Theridiidae, Amaurobiidae, Araneidae, Hahniidae and Liocranidae. In bog habitats in northern Manitoba, Aitchison-Benell (1994) found that the dominant spider families were the Erigonidae, Lycosidae, Linyphiidae and Gnaphosidae. In his work in the north-central part of Alberta, Buddle (2001) found that species in the Lycosidae family were numerically dominant, representing $44 \%$ of the total catch, followed by species in the Linyphiidae family at $30 \%$ of the total catch. 


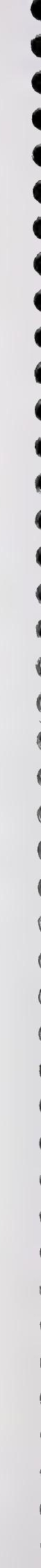


Figure 2. Spiders collected within four wildland parks in northeastern Alberta. Numbers of species represented per family and adult specimens collected per family are shown at the tops of the bars.

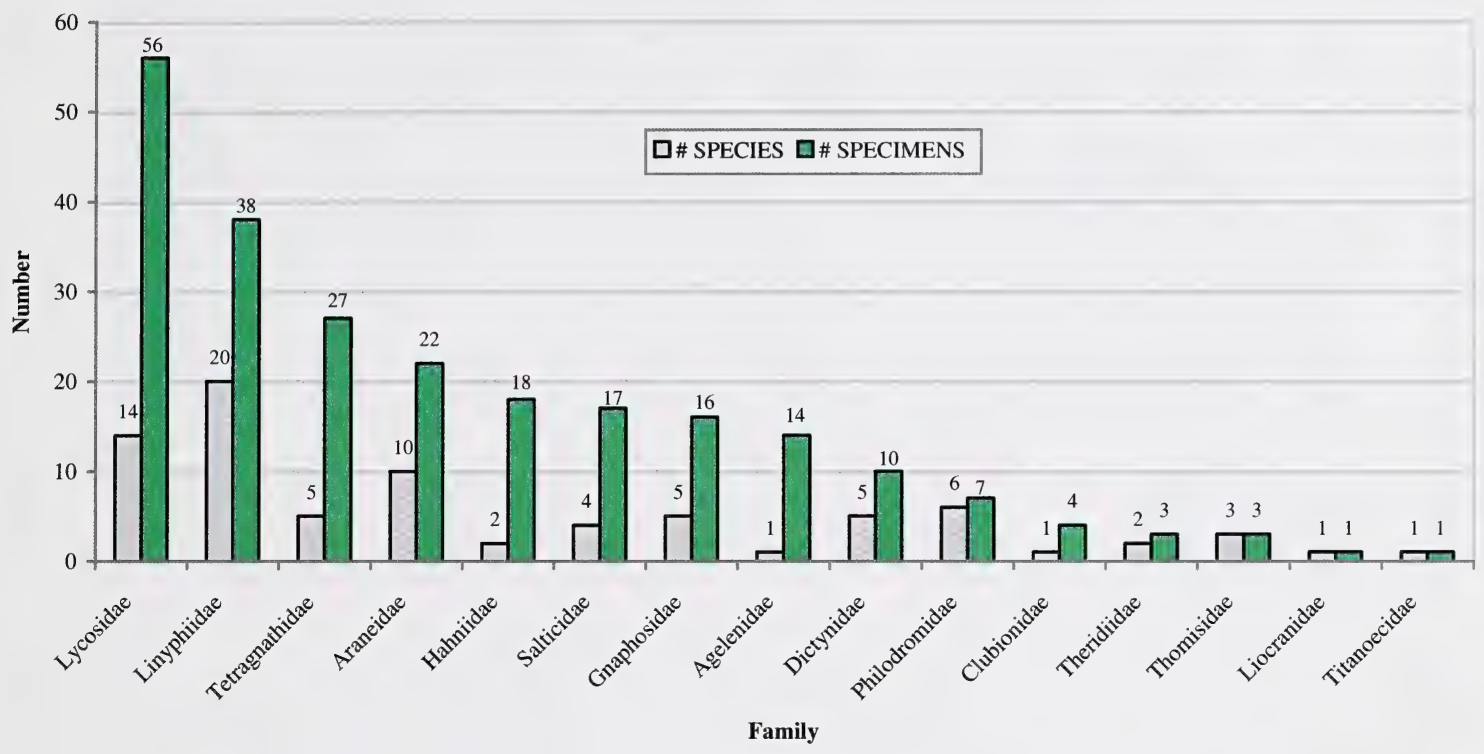

In this survey, the highest diversity of species within particular families were found in the Linyphiidae, Lycosidae and Araneidae (Table 1, Figure 2). In northern Manitoba, Aitchison and Sutherland (2000) found that the families Lycosidae, Gnaphosidae and Erigonidae had the greatest species diversity. Buddle (2001) found the highest species diversity in the Linyphiidae family.

In total, 233 adult specimens (125 females, 108 males) were collected (Table 2). Most of the specimens that were collected were from common, wide ranging species. The proportion of males and females in the total catch was not significantly different. However, there were considerably more agelenid, linyphiid and salticid males in the catch than females (Table 2). Approximately 50\% (233/466) of the total number of specimens collected was comprised of immatures (Table 2).

The five species that were most commonly collected were Pardosa groenlandica (18 specimens), Neoantistea magna (17 specimens), Tetragnatha versicolor (14 specimens), Agelenopsis utahana (14 specimens) and Eris militaris (11 specimens) (Figure 3). In northern Manitoba, Aitchison and Sutherland (2000) also found Agelenopsis utahana to be a dominant ground-dwelling species. For most species, only a few specimens were obtained. Approximately $41 \%(96 / 233)$ of the specimens were from species for which fewer than four specimens were collected (Figure 4). Unfortunately, these results cannot be interpreted to estimate species' rarity, because sampling effort was not controlled either temporally or spatially. Buddle (2001) also noted that many of the spider species he collected were uncommon or rare (e.g., 31 species were represented by only one individual). 

Table 2. Adult male, adult female and immature specimens in each of the 15 spider families represented in the four wildland parks of northeastern Alberta.

\begin{tabular}{|c|c|c|c|c|c|}
\hline FAMILY & No. immatures & No. females & No. males & $\begin{array}{c}\text { Total } \\
\text { (females + males) }\end{array}$ & $\begin{array}{c}\text { Percent } \\
(\text { female }+ \text { male }) / \text { total }\end{array}$ \\
\hline Agelenidae & 2 & 2 & 12 & 14 & 6.0 \\
\hline Araneidae & 8 & 17 & 5 & 22 & 9.4 \\
\hline Clubionidae & 4 & 0 & 0 & 0 & 0 \\
\hline Dictynidae & 5 & 5 & 5 & 10 & 4.3 \\
\hline Gnaphosidae & 10 & 8 & 8 & 16 & 6.9 \\
\hline Hahniidae & 2 & 15 & 3 & 18 & 7.7 \\
\hline Liocranidae & 0 & 0 & 1 & 1 & 0.4 \\
\hline Linyphiidae & 9 & 13 & 25 & 38 & 16.3 \\
\hline Lycosidae & 139 & 34 & 22 & 56 & 24.0 \\
\hline Philodromidae & 14 & 4 & 3 & 7 & 3.0 \\
\hline Salticidae & 12 & 3 & 14 & 17 & 7.3 \\
\hline Tetragnathidae & 3 & 21 & 6 & 27 & 11.6 \\
\hline Theridiidae & 4 & 1 & 2 & 3 & 1.3 \\
\hline Thomisidae & 21 & 2 & 1 & 3 & 1.3 \\
\hline Titanoecidae & 0 & 0 & 1 & 1 & 0.4 \\
\hline TOTAL & 233 & 125 & 108 & 233 & 100 \\
\hline
\end{tabular}

Figure 3. Spider species for which four or more specimens were collected.

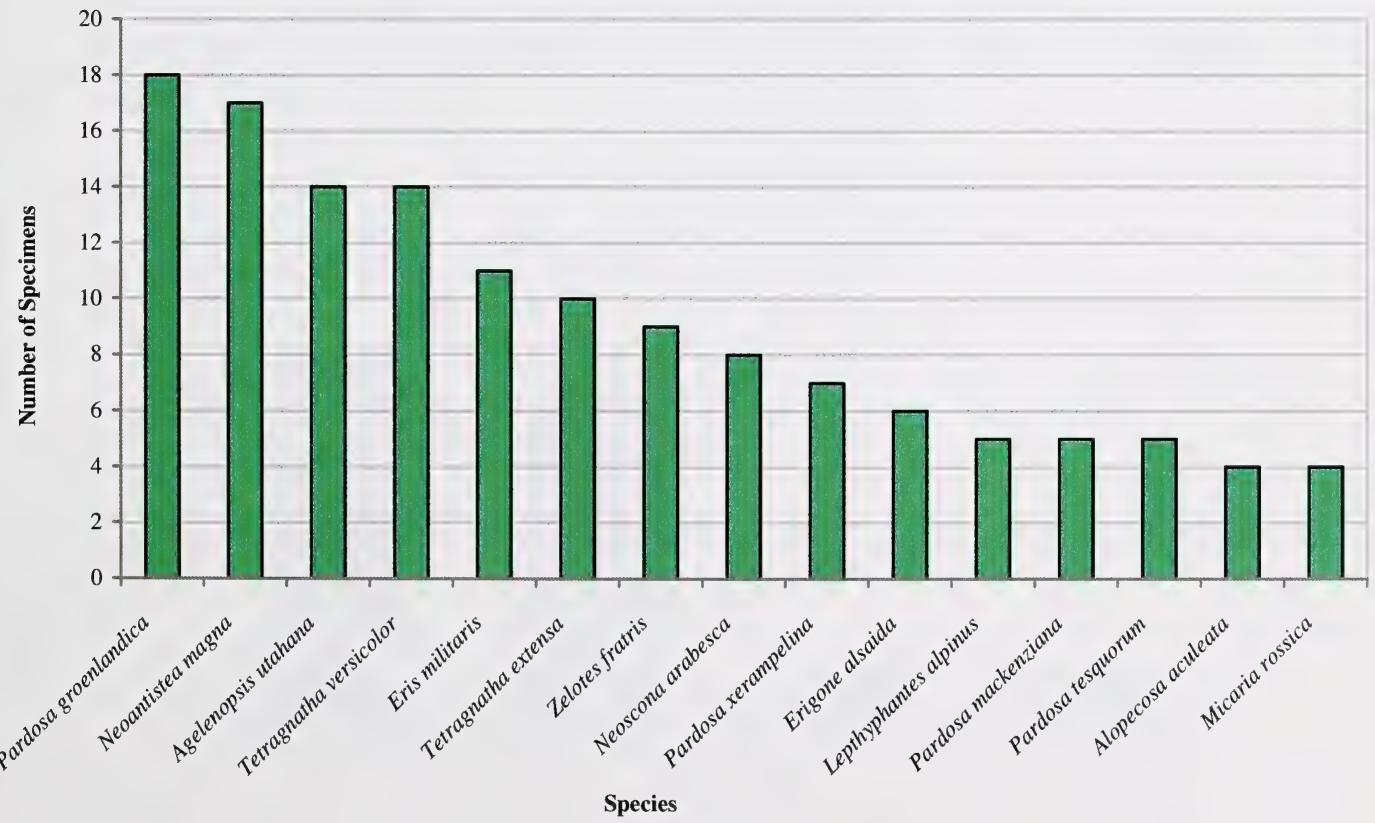



Figure 4. The number of adult specimens collected per species. The species for which four or more specimens were collected are separated from the "Others" that had fewer than four specimens collected per species.

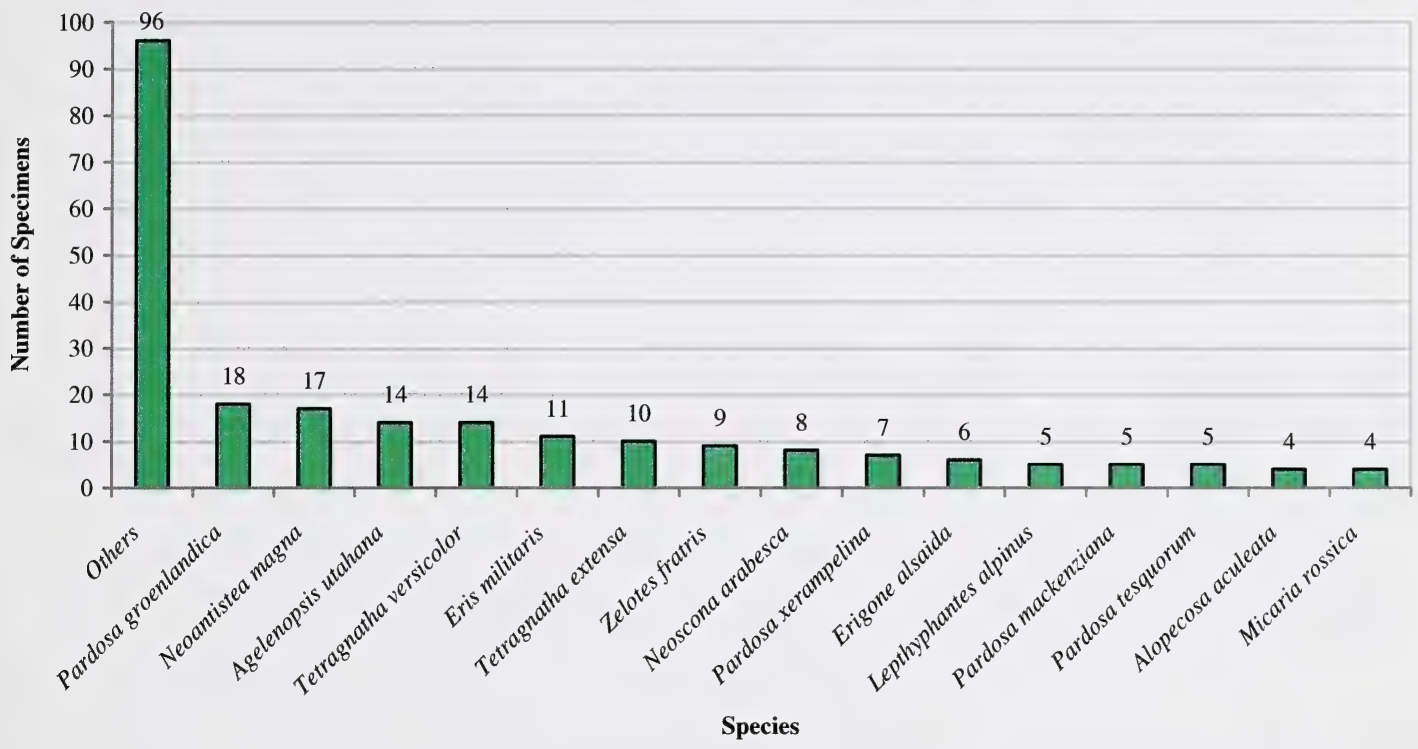

Some of the wildland parks were better sampled than others. The majority of the specimens were collected from Fidler-Greywillow Wildland (111/233 or 47.6\%) and La Butte Creek Wildland $(99 / 233$ or $42.4 \%$ ) (Figure 5).

Figure 5. The number of adult specimens collected within each of the wildland parks (numbers collected are shown at the tops of the columns).

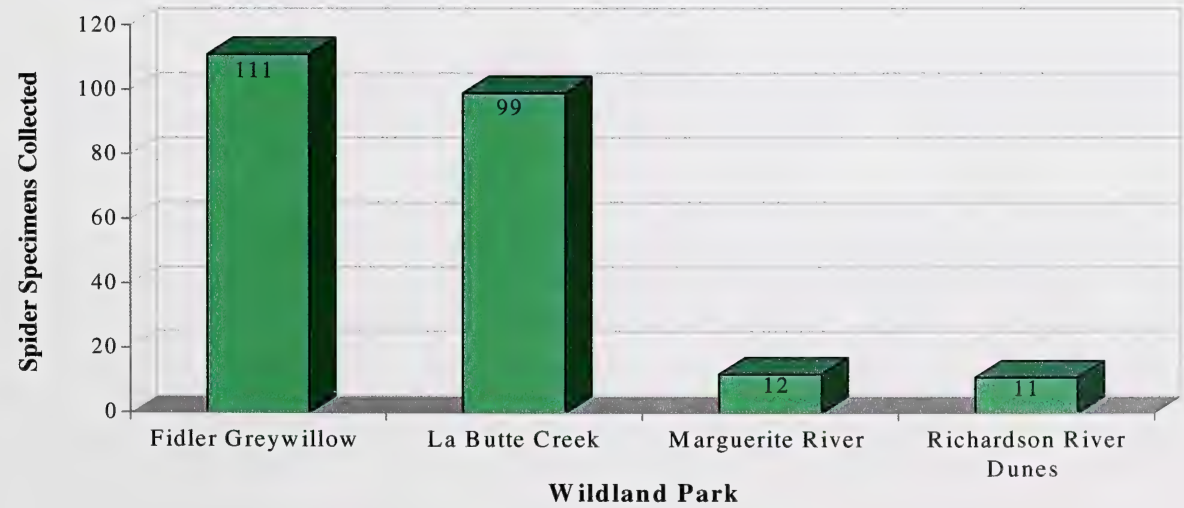



The specimen records from this study have contributed to what is known about the current ranges of these species. There were several notable finds, including the following:

- Schizocosa minnesotensis. This species is normally a grassland species that occurs on the Great Plains from New Mexico north to the prairie and parkland regions of Saskatchewan and Alberta, and in the inter-montane valleys of the west from Oregon to southern British Columbia. Unexpected records of this wolf spider in the Richardson River Dunes Wildland area represent a newly discovered disjunct population for northeastern Alberta. One female of the species was collected at the Richardson Fire Tower and one male from the Gogo Lake area south of Lake Athabasca (Appendix 1, p17). Another disjunct population of the species is known from the southern Yukon on dunes and sagebrush covered slopes in the Mount Wallace and Sheep Mountain areas (Dondale et al. 1997).

- Argenna prominula. This species is widespread but rarely collected. It has been reported from the western mountains (New Mexico to the Yukon) and from northern Manitoba. There are no published records for Alberta. One male was collected from a bog in La Butte Creek Wildland Park.

- Philodromus mysticus. This species is widely distributed in the boreal from Newfoundland to British Columbia, but is rarely collected. One male was collected in the Ft. Chipewyan Winter Road area south of Lake Athabasca at km 130 (UTM12, 502771 E, 6454034 N), near Maybelle River Wildland Park.

- Dictyna phylax. This species is widespread but rarely collected. There are no published records for Alberta. One male and one female were collected from Marguerite River Wildland Park (UTM12, 539695 E, 6396747 N).

- Erigone alsaida. There are no published records of this species for Alberta. Six males were collected from the Fidler Point area of Fidler / Greywillow Wildland Park on 11 June 2001.

- Pelecopsis bishopi. Records of this species from the shield area significantly extend its range to the west and north. It was previously reported from the eastern USA, westward to east-central Manitoba. During surveys in the shield, one male of the species was collected from La Butte Creek Wildland Park in July of 2001.

- Pelecopsis moesta. This is an eastern species previously found only as far west as Saskatchewan. One male was collected from the Fidler Point area on Lake Athabasca within Fidler-Greywillow Wildland Park.

- Habronattus captiosus. The known distribution of this species is unusual. It has previously been reported from the American Midwest (Michigan and Minnesota), and from the foothills and front ranges of the Rockies from southwestern Alberta to the southern Yukon. One male was collected near the Richardson River Dunes Wildland Park at the Richardson Fire Tower Airstrip (UTM12, 497900 E, 6415700 N). Another male was collected from the Fidler Point area on Lake Athabasca within Fidler-Greywillow Wildland Park. These are significant additions to its known distribution. 


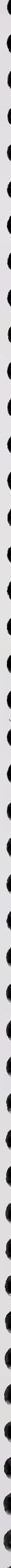


- Grammonota sp. The specimens obtained in the shield appear to represent a new species belonging to the Grammonota pictilis complex. One female specimen was collected from the Fidler Point area on Lake Athabasca and one male from the La Butte Creek Wildland Park, both in July of 2001.

- Dictyna arundinacea. There are no published records of this species for Alberta. One male and one female were collected from the Fort Chipewyan Road \& Richardson River area (UTM12, 498410 E, 6429588 N ), near Richardson River Dunes Wildland Park. One male was collected from Marguerite River Wildland Park (UTM12, 539695 E, 6396747 N).

- Thanatus formicinus. The specimens collected in the shield are a northern extension of the species' known range. Previous northernmost records in western Canada were from Baptiste Lake, Alberta and near Cumberland House, Saskatchewan. During the survey in 2001, one female was collected from La Butte Creek Wildland Park and one female from the Fidler Point area on Lake Athabasca.

Appendix 1 and 2 outline in more detail where and when each species was collected as well as some notes on their distribution and habitat. Since sample size was quite small (23 specimens) for spiders collected in the 'boreal forest parks' (i.e., Richardson River Dunes, Marguerite River), little attempt was made in the discussion above to analyze the data from the point of view of 'shield parks' versus 'boreal forest parks'.

\section{Conclusion}

The field program of 2000 and 2001 for wildland parks in northeastern Alberta included spiders as part of its biophysical inventory, but ancillary to other taxa. Spider specimens were collected but sampling effort was not standardized. Nevertheless, the specimens obtained were valuable in that they (a) improved our knowledge of species' ranges and life histories / phenologies in Alberta and Canada, (b) potentially contributed to the discovery of new undescribed species, and (c) added to our understanding of the overall biodiversity of these areas.

Given the season in which the field program occurred (i.e., June, July and August) and the length of time spent at each wildland park, a number of species were likely missed. Better data would have been obtained if more extensive sampling had occurred over a broader time period and over more than one field season. This would allow more accurate conclusions to be drawn relative to the abundance and distribution of species in these areas. 


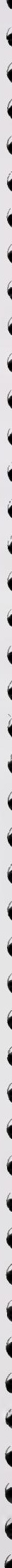




\section{Acknowledgements}

Without the help and support of dedicated and enthusiastic people, this report would not have been possible. Thanks are extended to Robert Holmberg and Sherri Fownes who reviewed and provided helpful comments on this report. Their input was appreciated. Robert Holmberg also provided suggestions in terms of inventory procedures and methodology.

Thanks are also due to the Parks and Protected Area's staff of the Northeast Boreal Region, and in particular, Ted Johnson and Jennifer Gammon, for their work in making it possible to access sites and conduct field work in the wildland parks of northeastern Alberta. Without that logistic support, the inventory project would not have happened. Thanks also to the volunteers who were part of the field crew; they supplied specimens and provided support in the field.

The specimens will be housed in the E.H. Strickland Entomological Museum at the University of Alberta. We extend our appreciation to Felix Sperling and Danny Shpeley for their support in accepting and housing those specimens. 



\section{Literature Cited}

Aitchison-Benell, C.W. 1994. Bog arachnids (Araneae, Opiliones) from Manitoba taiga. Memoirs of the Entomological Society of Canada 169: 21-31.

Aitchison, C.W. and G.D. Sutherland. 2000. Diversity of forest upland arachnid communities in Manitoba taiga (Araneae, Opiliones). Canadian Field Naturalist 114(4): 636-651.

Alberta Environmental Protection. 1998. Natural Regions and Subregions of Alberta. 1:1,000,000 scale map. Produced by Resource Data Division.

Bennett, R.G. 1999. Canadian spider diversity and systematics. Newsletter of the Biological Survey of Canada (Terrestrial Arthropods) Volume 18 No.1.

URL: http://www.biology.ualberta.ca/esc.hp/bsc/news18 1/spider.htm

Buckle, D.J. and R. G. Holmberg. 2001. Araneae of Alberta and Saskatchewan, Canada.

International Society of Arachnology. Abstract from the 2001 International Congress of Arachnology in Badplaas, South Africa.

Buddle, C. 2000. Alberta Spiders. URL: http://www.biology.ualberta.ca/uasm/alta.spiders.htm

Buddle, C.M. 2001. Spider communities in boreal mixed-wood forests of Alberta: succession, species interactions and habitat utilization. Ph.D. Thesis. Department of Biological Sciences, University of Alberta, Edmonton. 203pp.

Buddle, C.M. and D.P. Shorthouse. 2000. Jumping Spiders of Canada. Department of Biological Sciences, University of Alberta, Edmonton, Alberta. http://www.ualberta.ca/ dps1/Salticidae.html

Dondale, C.D., J.H. Redner, and Y.M Marusik. 1997. Spiders (Araneae) of the Yukon. pp. 73-113 in H.V. Danks and J.A. Downes (Eds.), Insects of the Yukon. Biological Survey of Canada (Terrestrial Arthropods), Ottawa. 1034 pp.

Finnamore, A.T., N.N. Winchester and V.M. Behan-Pelletier. 2001. Protocols for measuring biodiversity: arthropod monitoring in terrestrial ecosystems. URL: http://eqbdqe.cciw.ca/eman/ecotools/protocols/terrestrial/arthropods/pan.html

Shorthouse, D. and C. Buddle. May 2000. Who are the Canadian arachnologists? Canadian Arachnologist 1:3. 


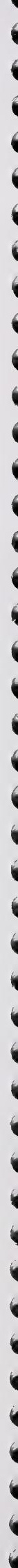




\section{Personal Communications}

Holmberg, Robert. 2001. Mailing address: Centre for Science; Athabasca University; 1 University Drive; Athabasca, AB; T9S 3A3. Email address: robert@ athabascau.ca

Leech, Robin. 2001. Mailing address: Biological Sciences; Northern Alberta Institute of Technology; 11762 - 106 St.; Edmonton, AB.; T5G 2R1. Email address: releech@telusplanet.net 


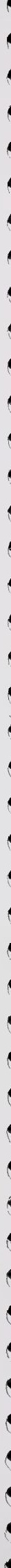


AGELENIDAE (funnel web weavers)

Agelenopsis utahana (Chamberlin and Ivie). Widespread. Reaches its northern limits in Alaska, Yukon Territory and Northwest Territories. Five males and two females were collected from La Butte Creek WP. Seven males and one immature were collected from the Fidler Point area of Lake Athabasca.

ARANEIDAE (orb weavers)

Araneus marmoreus (Clerck). Holarctic. Widespread. Webs usually on shrubs. One female was collected in La Butte Creek WP and one female in Fidler/Greywillow WP.

Araneus trifolium (Hentz). Widespread. Webs in grass or herbs. One male was collected in La Butte Creek WP.

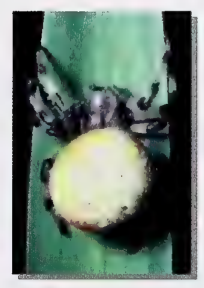

Araneus trifolium (female)

Photo by D. Buckle

Araneus nordmanni (Thorell). Holarctic. Widespread. Webs on trees. One female was collected in Fidler/Greywillow WP.

Cyclosa conica (Pallas). Widespread. Webs on conifers. One immature was collected in La Butte Creek WP.

Hypsosinga pygmaea (Sundevall). Holarctic. Widespread. Two females were collected in La Butte Creek WP.

Hypsosinga rubens (Hentz). Widespread. One female was collected in Marguerite River WP (UTM12, 539695 E, 6396747 N).

Larinioides patagiatus (Clerck). Holarctic. Widespread. Two females were collected on Bustard Island and one female in the Fidler Point area of Lake Athabasca.

Metepeira palustris (Chamberlin and Ivie). Boreal. Webs or grass or herbs. One male and two females were collected from La Butte Creek WP.

Neoscona arabesca (Walckenaer). Widespread. One male was collected from the Fidler Point area of Lake Athabasca. One male and six females were collected from La Butte Creek WP.

Zygiella nearctica (Gertsch). Northern North America, except west coast. Webs on trees. One male and two immatures were collected from the Fidler Point area of Lake Athabasca.

CLUBIONIDAE (sac spiders)

Clubiona sp. Four immatures were collected from the Fidler Point area of Lake Athabasca.

DICTYNIDAE (meshweb weavers)

Argenna prominula (Tullgren). Holarctic. Northern and montane. Widespread but rarely collected. Has been reported from the western mountains (New Mexico to Yukon Territory) and from northern Manitoba. There are no published records for Alberta. One male was collected from a bog in La Butte Creek WP. 


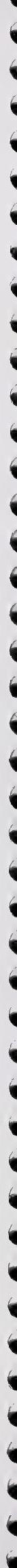


Dictyna annulipes (Blackwall). Holarctic. Northern and central North America. One male was collected from Bustard Island in Lake Athabasca.

Dictyna arundinacea (Linnaeus). Holarctic. Northern and montane. There are no published records for Alberta. One male and one female were collected from the Fort Chipewyan Road and Richardson River area (UTM12, 498410 E, 6429588 N ). One male was collected from Marguerite River WP (UTM12, 539695 E, 6396747 N).

Dictyna brevitarsus (Emerton). Northern and western North America. One female was collected from La Butte Creek WP.

Dictyna phylax (Gertsch and Ivie). Northern North America. Widespread but rarely collected. There are no published records for Alberta. One male and one female were collected from Marguerite River WP (UTM12, 539695 E, 6396747 N).

Dictyna sp. \#1. One female was collected from the Fort Chipewyan Road and Richardson River area (UTM12, $498410 \mathrm{E}, 6429588 \mathrm{~N}$ ).

Dictyna sp. \#2. One female was collected from Marguerite River WP (UTM12, 539695 E, 6396747 N).

GNAPHOSIDAE (ground spiders)

Gnaphosa parvula (Banks). Northern and central North America. One male and one female were collected from La Butte Creek WP.

Micaria aenea (Thorell). Holarctic. Northern. One female was collected from La Butte Creek WP. Micaria rossica (Thorell). Holarctic. Occurs from Alaska south to California and New Mexico, and east to Manitoba. One male and three females were collected from the Fidler Point area on Lake Athabasca.

Zelotes fratris (Chamberlin). Widespread. Three males were collected from La Butte Creek WP. Three males and two females were collected from the Fidler Point area on Lake Athabasca. One female was collected from the Marguerite River area (UTM12, 535253 E, 6394577 N).

HAHNIIDAE (dwarf funnel spiders)

Neoantistea agilis (Keyserling). Widely distributed: northern boreal to southern USA. One female was collected from the Fidler Point area on Lake Athabasca.

Neoantistea magna (Keyserling). Widely distributed: Alaska to Florida. Seven females were collected on Bustard Island in Lake Athabasca; in addition, 3 females and 3 males were collected from the Fidler Point area on Lake Athabasca. Four females were collected from La Butte Creek WP.

LINYPHIIDAE (LINYPHIINAE) (sheetweb weavers)

Agyneta sp. \#1. This large genus is badly in need of revision. Many western species cannot be placed. One male and one female were collected from La Butte Creek WP.

Bathyphantes pallidus (Banks). Northern and central North America. One male was collected from La Butte Creek WP.

Lepthyphantes complicatus (Emerton). Holarctic. Northern. One male was collected on Bustard Island in Lake Athabasca.

Lepthyphantes alpinus (Emerton). Northern. Three males and one female were collected from La Butte Creek WP. One female was collected on Bustard Island in Lake Athabasca.

Microneta viaria (Blackwall). Holarctic. Widespread. Two males were collected on Bustard Island in Lake Athabasca.

Pityohyphantes subarcticus (Chamberlin and Ivie). A boreal species. Webs on conifers. One male and one female were collected from the Fidler Point area on Lake Athabasca. 


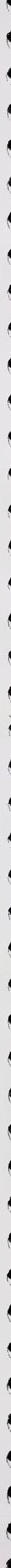




\section{LINYPHIIDAE (ERIGONINAE) (dwarf weavers)}

Ceraticelus sp. \#1, fissiceps group. One female was collected from La Butte Creek WP.

Ceratinopsis stativa (Simon). Holarctic. Northern. One male was collected from La Butte Creek

WP and one male from the Fidler Point area on Lake Athabasca.

Diplocentria bidentata (Emerton). Holarctic. Northern, south in the Rockies to Utah. Two males were collected from La Butte Creek WP.

Diplocephalus subrostratus (O.P.-Cambridge). Holarctic. Northern. One female was collected from La Butte Creek WP.

Dismodicus alticeps (Chamberlin and Ivie). Holarctic. Northern. One female was collected from Marguerite River WP (UTM12, 539695 E, 6396747 N).

Erigone blaesa (Crosby and Bishop). Widespread. One male was collected from La Butte Creek WP.

Erigone alsaida (Crosby and Bishop). Six males were collected from the Fidler Point area on Lake Athabasca. There are no published records of this species for Alberta.

Gnathonarium famelicum (Keyserling). Northern. Two females were collected from the Fidler Point area on Lake Athabasca.

Grammonota sp. \#1. Apparently an undescribed species belonging to the pictilis complex. One female specimen was collected from the Fidler Point area on Lake Athabasca and one male from the La Butte Creek area, both in July of 2001.

Pelecopsis bishopi (Kaston). A significant range extension west and north. Previously reported from the eastern USA, west to east-central Manitoba. One male was collected from La Butte Creek WP in July of 2001.

Pelecopsis moesta (Banks). An eastern species previously found as far west as Saskatchewan. One male was collected from the Fidler Point area on Lake Athabasca.

Walckenaeria communis (Emerton). Northern and central North America. Two females were collected from La Butte Creek WP.

Walckenaeria directa (O.P.-Cambridge). Widespread. One female was collected from La Butte Creek WP.

Maso sundevalli (Westring 1851). Two males were collected from La Butte Creek WP.

LIOCRANIDAE (no common name)

Agroeca ornata (Banks). Widespread. One male was collected from La Butte Creek WP.

LYCOSIDAE (wolf spiders)

Alopecosa aculeata (Clerck). Holarctic: Alaska to Arizona. One female was collected from Marguerite River WP (UTM12, 539695 E, 6396747 N), one female from La Butte Creek WP, and two males from Bustard Island in Lake Athabasca. Two immatures were collected from the Fidler Point area of Lake Athabasca and two immatures from La Butte Creek WP.

Arctosa alpigena (Doleschall). Holarctic: arctic, alpine, boreal. One female was collected from La Butte Creek WP.

Hogna frondicola (Emerton). Widely distributed: northern boreal to southern USA. One male and one female were collected from the Fidler Point area of Lake Athabasca.

Pardosa concinna (Thorell). Open habitats, from low arctic to northern Great Plains. One male was collected from the Gogo Lake area south of Lake Athabasca (UTM12, 497800 E, 6415500 N) and one female from La Butte Creek WP.

Pardosa fuscula (Thorell). Holarctic: low arctic to northern and alpine USA. One female was collected from La Butte Creek WP. 


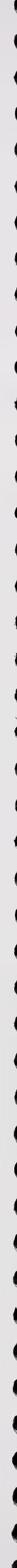


Pardosa groenlandica (Thorell). Holarctic: arctic to boreal. One male, four females, and sixteen immatures were collected from La Butte Creek WP. Four males, two females, and 53 immatures were collected from the Fidler Point area on Lake Athabasca. Five males, two females, and 29 immatures were collected from Bustard Island in Lake Athabasca.

Pardosa hyperborea (Thorell). Holarctic: low arctic to boreal. One male was collected from La Butte Creek WP. Two females and eight immatures were collected from the Fidler Point area on Lake Athabasca.

Pardosa mackenziana (Keyserling). Low arctic to northern and western montane USA. Two females were collected from La Butte Creek WP. One male and one female were collected from the Fidler Point area on Lake Athabasca. One male was collected from Marguerite River WP (UTM12, 539695 E, 6396747 N).

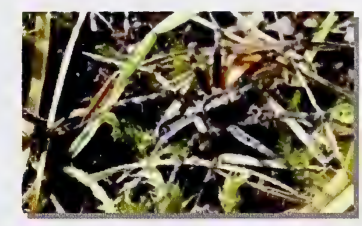

Pardosa mackenziana

Photo by C. Buddle

Pardosa moesta (Banks). Northern boreal to mid-USA. Two females were collected from La Butte Creek WP. One male was collected from Gogo Lake south of Lake Athabasca (UTM12, $497800 \mathrm{E}, 6415500 \mathrm{~N})$.

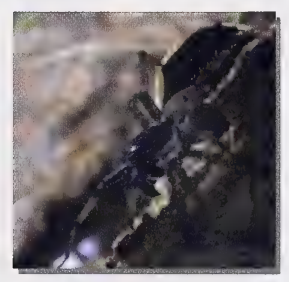

Pardosa moesta

Photo by C. Buddle

Pardosa tesquorum (Odenwall). Holarctic: low arctic to northern USA. Three males and one female were collected from La Butte Creek WP. One female was collected from the Fidler Point area on Lake Athabasca.

Pardosa xerampelina (Keyserling). Widely distributed: northern boreal to southern USA. Six females were collected from La Butte Creek WP and one female from Bustard Island in Lake Athabasca.

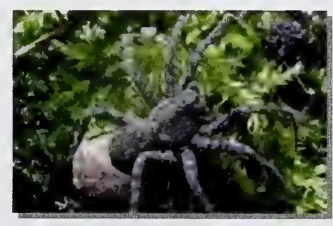

Pardosa xerampelina

Photo by C. Buddle

Pirata piraticus (Clerck). Holarctic: low arctic to southern USA. One female was collected from the Fidler Point area on Lake Athabasca. 

Schizocosa minnesotensis (Gertsch). One female of the species was collected at the Richardson Fire Tower Airstrip (UTM12, 497900 E, $6415700 \mathrm{~N}$ ) and one male from the Gogo Lake area south of Lake Athabasca (UTM12, 497800 E, $6415500 \mathrm{~N}$ ). These specimens represent a newly discovered disjunct population in northeastern Alberta. Another disjunct population is known from the southern Yukon on dunes and sagebrush covered slopes on Mt. Wallace and Sheep Mountain. This species is normally a grassland species occurring on the Great Plains from New Mexico north to the prairie and parkland regions of Saskatchewan and Alberta, and in the intermontane valleys of the west from Oregon to southern British Columbia.

Trochosa pratensis (Emerton). Northern boreal to southern USA. Considered by some to be a synonym of the palaearctic Trochosa terricola (Thorell). One immature was collected from La Butte Creek WP. Two females and one immature were collected from the Fidler Point area on Lake Athabasca.

PHILODROMIDAE (philodromid crab spiders)

Philodromus cespitum (Walckenaer). Holarctic. Widespread. On shrubs. One female was collected from La Butte Creek WP and one female from the Fidler Point area on Lake Athabasca.

Philodromus histrio (Latrielle). Holarctic. Widespread. Open, usually dry grassland, habitats. One immature was collected from La Butte Creek WP and three immatures from the Fidler Point area on Lake Athabasca.

Philodromus mysticus (Dondale and Redner). Widely distributed in the boreal from Newfoundland to British Columbia, but rarely collected. One male was collected in the Ft. Chipewyan Winter Road area south of Lake Athabasca at km 130 (UTM12, 502771 E, 6454034 N).

Philodromus placidus (Banks). Widespread from the northern boreal south to Mexico. Occurs on conifer foliage. One male was collected from La Butte Creek WP and one from the Fidler Point area on Lake Athabasca.

Thanatus formicinus (Clerck). Range extension north. Previous northernmost records in western Canada were Baptiste Lake, Alberta and near Cumberland House, Saskatchewan. One female was collected from La Butte Creek WP and one female from the Fidler Point area on Lake Athabasca.

SALTICIDAE (jumping spiders)

Eris militaris (Hentz). Widespread. Found on shrubs and trees. Five males, one female and six immatures were collected from La Butte Creek WP. One female was collected from Gogo Lake south of Lake Athabasca (UTM12, 497800 E, $6415500 \mathrm{~N}$ ). Three males and one immature were collected from the Fidler Point area on Lake Athabasca. One female was collected from Marguerite River WP (UTM12, 539695 E, 6396747 N).

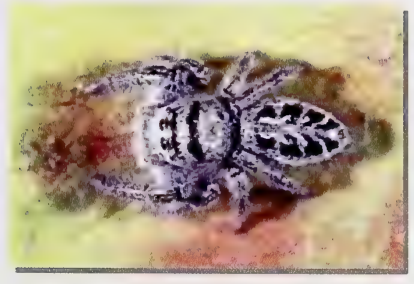

Eris militaris (female) Photo by D. Buckle

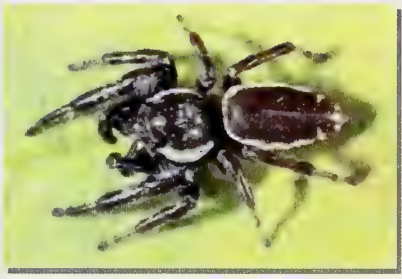

Eris militaris (male) Photo by D. Buckle 

Habronattus captiosus (Gertsch). What is known of this species' distribution is peculiar. It has previously been reported from the American Midwest (Michigan, Minnesota), and from the foothills and front range of the Rockies from southwestern Alberta to the southern Yukon. Buddle and Shorthouse (2000) report the species from the Yukon, British Columbia, Manitoba and Alberta. One male was collected from the Richardson Fire Tower Airstrip (UTM12, 497900 E, 6415700 N). One male was collected from the Fidler Point area on Lake Athabasca. Pelegrina flavipedes (Peckham and Peckham). Northern and central North America. Occurs on conifers. One male was collected from the Richardson Fire Tower Airstrip (UTM12, 497900 E, $6415700 \mathrm{~N})$.

Phidippus purpuratus (Keyserling). Widespread. Three males were collected from the Fidler Point area on Lake Athabasca.

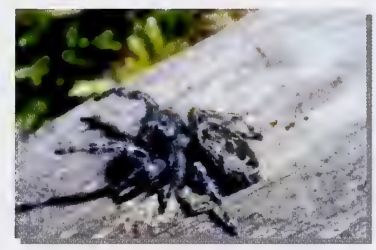

Phidippus sp.

Photo by C. Buddle

TETRAGNATHIDAE (long-jawed orb weavers)

Tetragnatha caudata (Emerton). Widespread. One female was collected from La Butte Creek WP.

Tetragnatha dearmata (Thorell). Holarctic. Boreal. One male was collected from the Fidler Point area on Lake Athabasca.

Tetragnatha shoshone (Levi). Widespread in northern North America. One male was collected from the Fidler Point area on Lake Athabasca.

Tetragnatha extensa (Linnaeus). Holarctic. Boreal/Hudsonian. One male and four females were collected from La Butte Creek WP. Five females were collected from the Fidler Point area on Lake Athabasca.

Tetragnatha versicolor (Walckenaer). Widespread. On shrubs and trees. One male and three females were collected from La Butte Creek WP. Two males and eight females were collected from the Fidler Point area on Lake Athabasca.

THERIDIIDAE (scaffold web spiders or cobweb weavers)

Theridion differens (Emerton). Widespread. Two males and three immatures were collected from Marguerite River WP (UTM12, 539695 E, 6396747 N).

Theridion pictum (Walckenaer). Holarctic. Widespread. One female was collected from the Fidler Point area on Lake Athabasca.

THOMISIDAE (thomisid crab spiders)

Misumena vatia (Clerck). Holarctic. Widespread. Five immatures were collected from La Butte Creek WP and two immatures from Marguerite River WP (UTM12, 539695 E, 6396747 N).

Ozyptila sincera canadensis (Dondale and Redner). Boreal. One female was collected from the Fidler Point area on Lake Athabasca.

Xysticus emertoni (Keyserling). Widespread. One female was collected from La Butte Creek WP and one male from the Fidler Point area on Lake Athabasca.

TITANOECIDAE (no common name)

Titanoeca silvicola (Chamberlin and Ivie). Northern and western North America. One male was collected from La Butte Creek WP. 


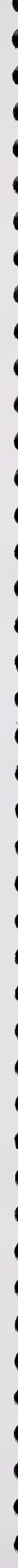




\begin{tabular}{|c|c|c|c|c|c|c|c|c|c|c|c|c|c|c|c|c|c|}
\hline \multirow{12}{*}{ 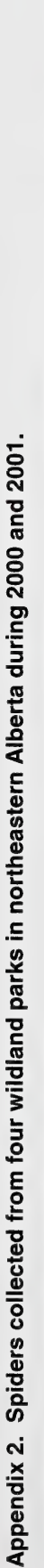 } & 音 & $\stackrel{m}{\Delta}$ & $\stackrel{m}{\Delta}$ & $\stackrel{m}{\Delta}$ & $\stackrel{m}{a}$ & $\stackrel{m}{\Delta}$ & $\stackrel{m}{a}$ & $\stackrel{\infty}{0}$ & 睘 & $\stackrel{m}{\Delta}$ & 睘 & $\stackrel{\text { mo }}{\text { ô }}$ & $\stackrel{\text { m }}{\Delta}$ & 䤧 & $\stackrel{\infty}{0}$ & $\stackrel{\infty}{0}$ & $\stackrel{m}{\Delta}$ \\
\hline & $\begin{array}{l}\frac{\grave{o}}{\mathrm{~d}} \\
\frac{\mathrm{\omega}}{\overline{0}} \\
\mathrm{o}\end{array}$ & 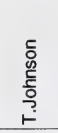 & 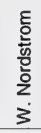 & 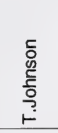 & 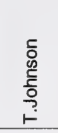 & 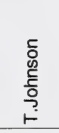 & 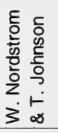 & 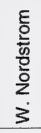 & 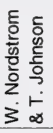 & 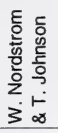 & 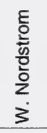 & 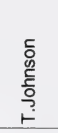 & 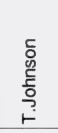 & 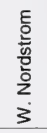 & 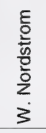 & 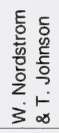 & 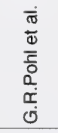 \\
\hline & 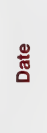 & 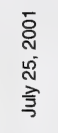 & $\begin{array}{l}\overline{8} \\
\frac{1}{3} \\
\overline{5} \\
\overline{0}\end{array}$ & 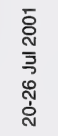 & 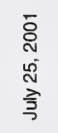 & 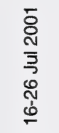 & $\begin{array}{l}\bar{D} \\
\bar{N} \\
\overline{5} \\
\overline{1} \\
\bar{b}\end{array}$ & 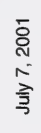 & $\begin{array}{l}\overline{\grave{d}} \\
\bar{N} \\
\overline{3} \\
\overline{0}\end{array}$ & $\begin{array}{l}\overline{8} \\
\bar{N} \\
\overline{5} \\
\overline{5} \\
\bar{i}\end{array}$ & 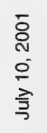 & 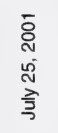 & 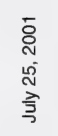 & 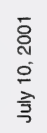 & $\begin{array}{l}\bar{o} \\
\frac{N}{3} \\
\overline{3} \\
\overline{0}\end{array}$ & 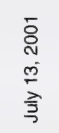 & 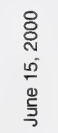 \\
\hline & 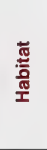 & & ठ্. & & & & 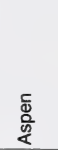 & $\begin{array}{l}\frac{5}{0} \\
\frac{0}{2} \\
\frac{2}{2}\end{array}$ & 당 & $\begin{array}{l}\text { क्ष } \\
\text { 웅 }\end{array}$ & $\frac{\grave{\mathrm{d}}}{\frac{\mathrm{o}}{\alpha}}$ & & & & రా & & 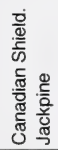 \\
\hline & 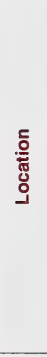 & 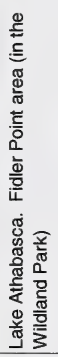 & 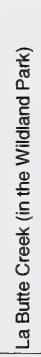 & 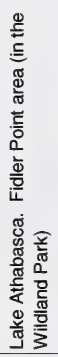 & 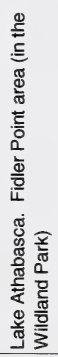 & 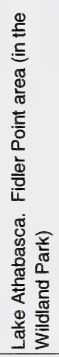 & 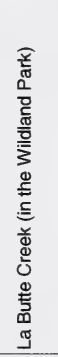 & 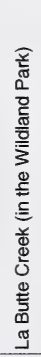 & 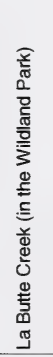 & 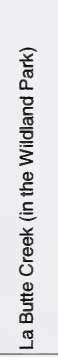 & 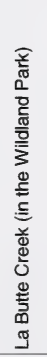 & 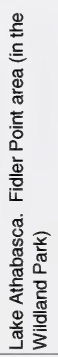 & 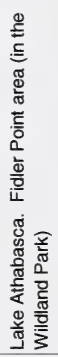 & 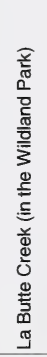 & 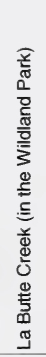 & 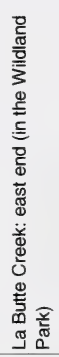 & 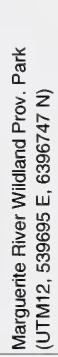 \\
\hline & 茲 ฮँ & & - & $\sigma$ & $\sim$ & - & $\sim$ & - & - & & & & & - & & & \\
\hline & 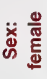 & & & & & & N & & & & - & - & - & & & $\sim$ & - \\
\hline & 芦 & & & & & & & & & & & & & & & & \\
\hline & 总总 & - & & & & & & & & - & & & & & - & & \\
\hline & 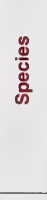 & 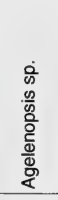 & 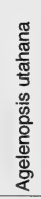 & 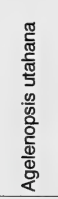 & 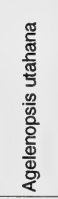 & 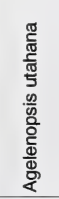 & 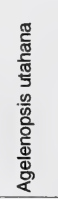 & 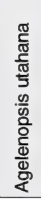 & 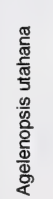 & 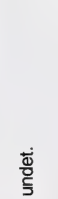 & 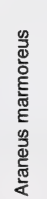 & 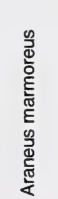 & 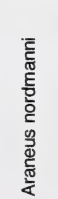 & 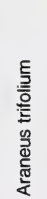 & 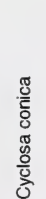 & 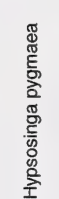 & 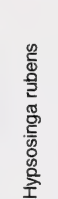 \\
\hline & 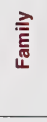 & 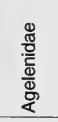 & 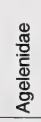 & 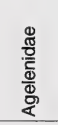 & 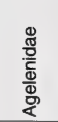 & 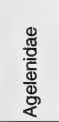 & 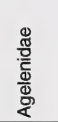 & 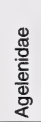 & 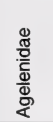 & 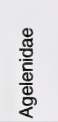 & 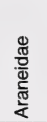 & 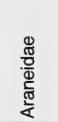 & $\begin{array}{l}\mathbb{\mathscr { J }} \\
\frac{\mathbb{\pi}}{\mathbb{W}} \\
\frac{\mathbb{w}}{4}\end{array}$ & 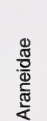 & 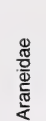 & 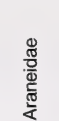 & 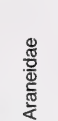 \\
\hline & 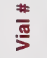 & $\bar{N}$ & $\stackrel{\infty}{\underline{\infty}}$ & กิ & $\bar{N}$ & ส & $\stackrel{\sim}{N}$ & \& & ని & $\stackrel{\sim}{\sim}$ & $\infty$ & $\bar{N}$ & $\bar{N}$ & $\stackrel{\varphi}{\prime}$ & $\stackrel{\infty}{\stackrel{\infty}{ }}$ & స & हे \\
\hline
\end{tabular}




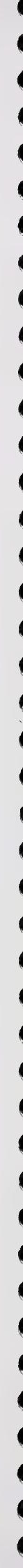




\begin{tabular}{|c|c|c|c|c|c|c|c|c|c|c|c|c|c|c|c|c|c|}
\hline \multirow[b]{13}{*}{$\frac{0}{4}$} & & & & & & & & & & & & & & & 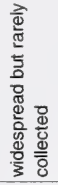 & & \\
\hline & 옴 & $\stackrel{\mathfrak{m}}{\mathrm{a}}$ & $\stackrel{m}{a}$ & $\stackrel{m}{\partial}$ & $\stackrel{m}{a}$ & $\stackrel{m}{a}$ & $\stackrel{m}{a}$ & $\stackrel{\text { mo }}{a}$ & $\stackrel{m}{a}$ & $\stackrel{m}{a}$ & $\stackrel{m}{a}$ & $\stackrel{m}{a}$ & $\stackrel{m}{a}$ & $\stackrel{m}{a}$ & $\stackrel{m}{0}$ & $\stackrel{m}{\Delta}$ & $\stackrel{m}{a}$ \\
\hline & $\begin{array}{l}\frac{\bar{o}}{\mathrm{~d}} \\
\overline{\bar{\omega}} \\
\bar{\delta}\end{array}$ & 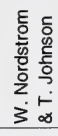 & 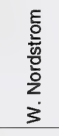 & 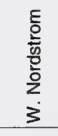 & 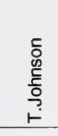 & 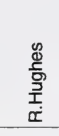 & 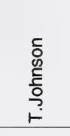 & 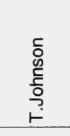 & 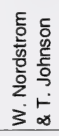 & 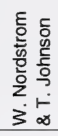 & 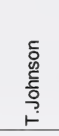 & 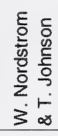 & 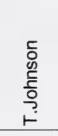 & 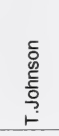 & 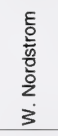 & 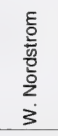 & 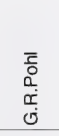 \\
\hline & $\frac{8}{\tilde{g}}$ & 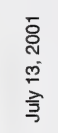 & 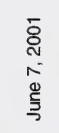 & 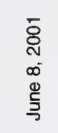 & 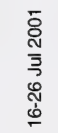 & 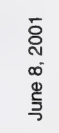 & 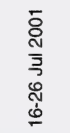 & 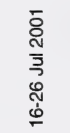 & 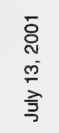 & 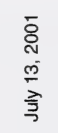 & 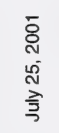 & 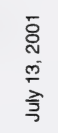 & 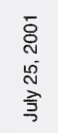 & 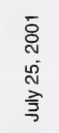 & $\begin{array}{l}\overline{0} \\
\bar{N} \\
\overline{3} \\
\overline{0}\end{array}$ & 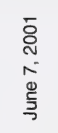 & 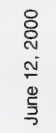 \\
\hline & & & & & & & 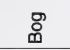 & & & & & & & & 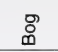 & & \\
\hline & 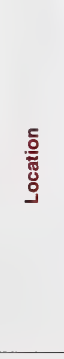 & 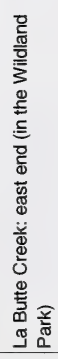 & 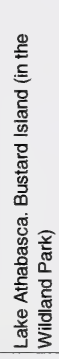 & 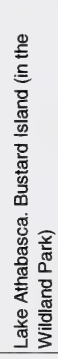 & 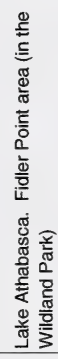 & 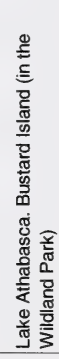 & 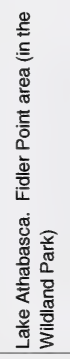 & 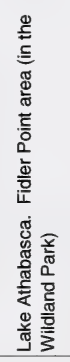 & 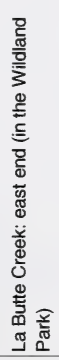 & 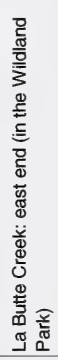 & 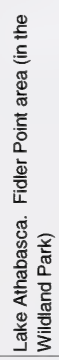 & 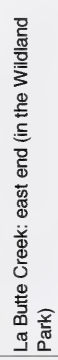 & 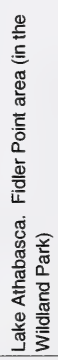 & 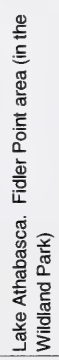 & 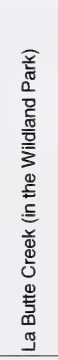 & 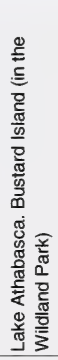 & 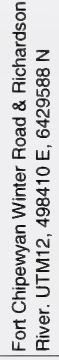 \\
\hline & 苂 & & & & & & & & & - & - & - & - & & - & - & - \\
\hline & 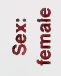 & & - & - & - & & & & & $N$ & & 0 & & & & & - \\
\hline & 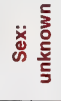 & & & & & & & & & & & & & & & & \\
\hline & 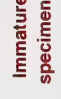 & - & & & & - & - & - & - & & & & $\sim$ & $\sigma$ & & & \\
\hline & $\begin{array}{l}\frac{\mathscr{\Xi}}{\mathbb{E}} \\
\frac{0}{2}\end{array}$ & 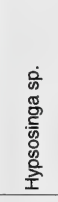 & 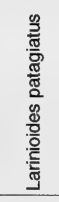 & 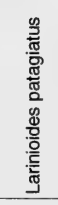 & 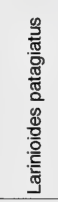 & 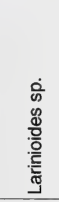 & 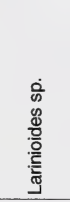 & 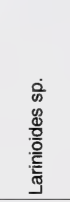 & 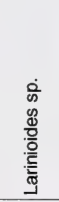 & 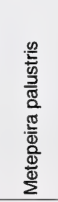 & 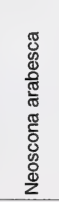 & 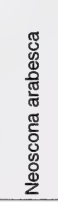 & 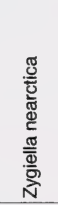 & 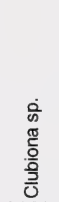 & 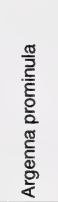 & 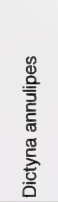 & 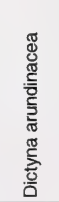 \\
\hline & 彥 & $\begin{array}{l}\frac{8}{\pi} \\
\frac{\pi}{6} \\
\frac{\pi}{5} \\
\frac{\pi}{4}\end{array}$ & $\begin{array}{l}\frac{\pi}{\pi} \\
\frac{\pi}{0} \\
\frac{\pi}{\pi} \\
\frac{\pi}{<}\end{array}$ & $\begin{array}{l}\frac{\mathbb{\pi}}{0} \\
\frac{0}{0} \\
\frac{\pi}{5} \\
\frac{\pi}{4}\end{array}$ & 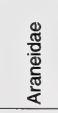 & $\begin{array}{l}\frac{\pi}{\pi} \\
\frac{\pi}{\pi} \\
\frac{\pi}{4} \\
\frac{\pi}{<}\end{array}$ & 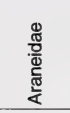 & 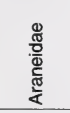 & 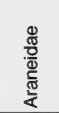 & $\begin{array}{l}\frac{\pi}{\pi} \\
\frac{\pi}{0} \\
\frac{\pi}{\pi} \\
\frac{\pi}{4}\end{array}$ & $\begin{array}{l}\frac{\pi}{\pi} \\
\frac{\pi}{0} \\
\frac{\pi}{\pi} \\
\frac{\pi}{4}\end{array}$ & $\begin{array}{l}\frac{\mathbb{\pi}}{0} \\
\frac{\pi}{0} \\
\frac{\pi}{5} \\
\frac{\pi}{4}\end{array}$ & $\begin{array}{l}\frac{8}{0} \\
\frac{\pi}{6} \\
\frac{\mathbb{6}}{4}\end{array}$ & 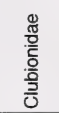 & 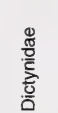 & 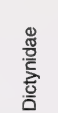 & 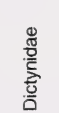 \\
\hline & 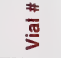 & న & $\sim$ & m & $\approx$ & \pm & $\stackrel{2}{=}$ & N & N & $\stackrel{N}{ }$ & $\bar{N}$ & N & $\bar{N}$ & $\bar{N}$ & $\stackrel{\infty}{=}$ & "̄ & ๑ \\
\hline
\end{tabular}





\begin{tabular}{|c|c|c|c|c|c|c|c|c|c|c|c|c|c|c|c|c|c|}
\hline \multirow{13}{*}{ 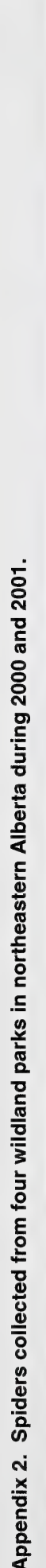 } & 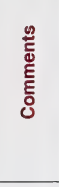 & & & 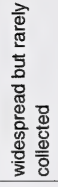 & & & & & & & & & & & & & \\
\hline & $\stackrel{\vec{a}}{\underline{Q}}$ & $\stackrel{m}{a}$ & $\stackrel{\mathscr{m}}{a}$ & $\stackrel{m}{a}$ & $\stackrel{m}{a}$ & $\stackrel{m}{a}$ & $\stackrel{m}{\vec{a}}$ & $\stackrel{m}{\text { Oे }}$ & 罢 & 罢 & $\stackrel{m}{a}$ & $\stackrel{m}{a}$ & $\stackrel{m}{a}$ & $\stackrel{\mathscr{m}}{\Delta}$ & $\stackrel{m}{a}$ & $\stackrel{m}{\circ}$ & $\stackrel{m}{3}$ \\
\hline & $\begin{array}{l}\stackrel{\bar{o}}{\circ} \\
\bar{\Xi} \\
\bar{\delta}\end{array}$ & 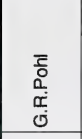 & 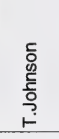 & $\begin{array}{l}\dot{\Phi} \\
\frac{\sigma}{\omega} \\
\frac{\sigma}{\sigma} \\
\frac{\sigma}{0} \\
\dot{\sigma} \\
\dot{\sigma}\end{array}$ & 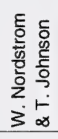 & 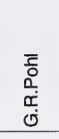 & $\begin{array}{l}\frac{\dot{\sigma}}{\sigma} \\
\frac{\sigma}{\sigma} \\
\frac{\bar{\sigma}}{0} \\
\frac{0}{0} \\
\tilde{\sigma} \\
0 \\
\end{array}$ & $\begin{array}{l}\bar{\Sigma} \\
0 \\
0 \\
\frac{0}{\pi} \\
0 \\
0\end{array}$ & 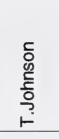 & 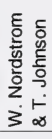 & 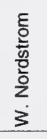 & 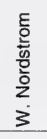 & 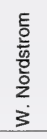 & 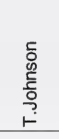 & 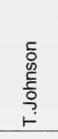 & 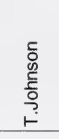 & 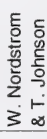 \\
\hline & ฐัँ & 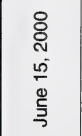 & 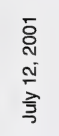 & $\begin{array}{l}8 \\
\stackrel{N}{1} \\
5 \\
05 \\
\stackrel{5}{5}\end{array}$ & 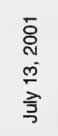 & 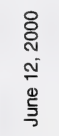 & 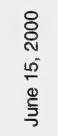 & 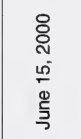 & 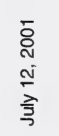 & $\begin{array}{l}\overline{0} \\
\bar{N} \\
\overline{3} \\
\overline{0}\end{array}$ & 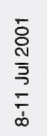 & $\begin{array}{l}\bar{\delta} \\
\frac{N}{3} \\
\overline{5} \\
\bar{\infty}\end{array}$ & $\begin{array}{l}\bar{\delta} \\
\frac{\bar{N}}{3} \\
\overline{3} \\
\bar{\infty} \\
\bar{\infty}\end{array}$ & 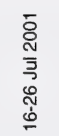 & 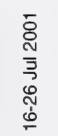 & 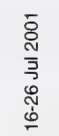 & $\begin{array}{l}\overline{0} \\
\frac{0}{n} \\
\overline{5} \\
\bar{\sigma} \\
\bar{b}\end{array}$ \\
\hline & 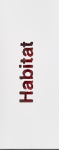 & 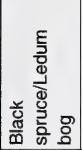 & 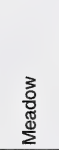 & 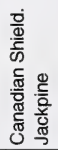 & & & 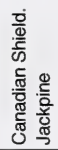 & 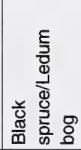 & 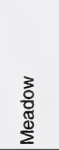 & $\begin{array}{l}\text { 임 } \\
\text { व. }\end{array}$ & & & & : & & & 酋 \\
\hline & 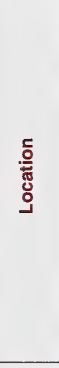 & 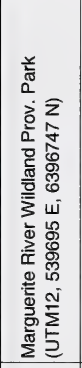 & 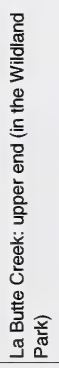 & 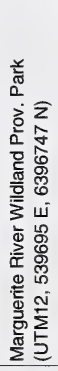 & 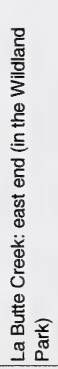 & 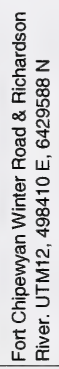 & 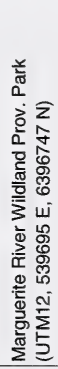 & 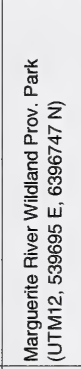 & 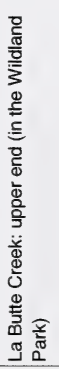 & 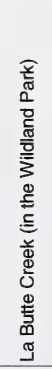 & 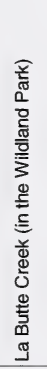 & 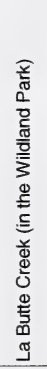 & 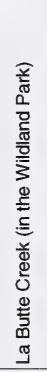 & 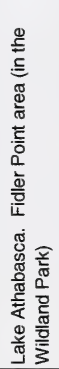 & 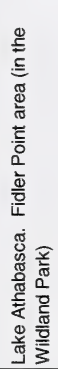 & 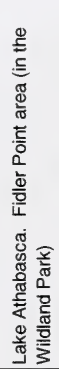 & 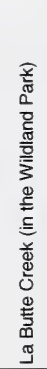 \\
\hline & 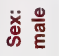 & - & & - & & & & & & & - & & & - & & & \\
\hline & 苂 & & - & - & & - & - & & & & - & & - & - & - & - & \\
\hline & 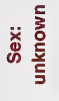 & & & & & & & & - & & & & & & & & \\
\hline & 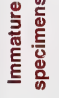 & & & & $\sigma$ & & & - & & - & & - & & & & & - \\
\hline & $\begin{array}{l}\frac{\mathscr{d}}{\mathbb{E}} \\
\frac{\mathrm{g}}{\infty}\end{array}$ & 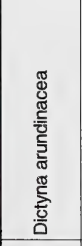 & 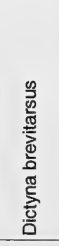 & 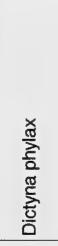 & 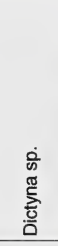 & 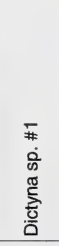 & 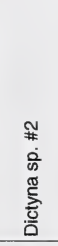 & 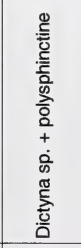 & 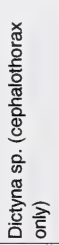 & 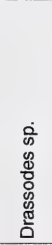 & 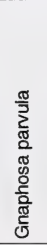 & 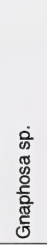 & 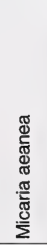 & 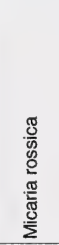 & 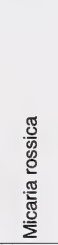 & 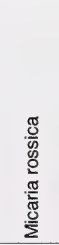 & 苟 \\
\hline & 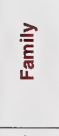 & 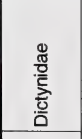 & 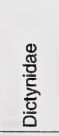 & 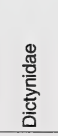 & 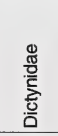 & 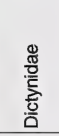 & 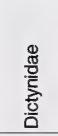 & 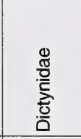 & 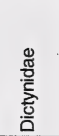 & 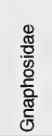 & 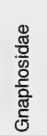 & $\begin{array}{l}\frac{\pi}{\pi} \\
\frac{\pi}{00} \\
\frac{0}{0} \\
\frac{0}{0} \\
\frac{0}{0}\end{array}$ & $\begin{array}{l}\frac{0}{0} \\
\frac{\pi}{00} \\
\frac{0}{0} \\
\frac{0}{0} \\
\frac{0}{0}\end{array}$ & $\begin{array}{l}\mathbb{8} \\
\frac{\mathbb{0}}{0} \\
\frac{0}{0} \\
\frac{0}{0} \\
\mathbb{E} \\
\mathbb{E}\end{array}$ & 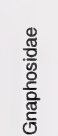 & 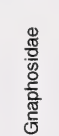 & 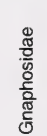 \\
\hline & 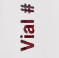 & 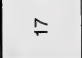 & N & ळ్ & స & ه & \& & 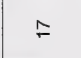 & $\hat{N}$ & ని & $\stackrel{\infty}{N}$ & $\stackrel{\infty}{N}$ & $\stackrel{\infty}{N}$ & $\stackrel{ }{\circ}$ & $\approx$ & న & 尺 \\
\hline
\end{tabular}





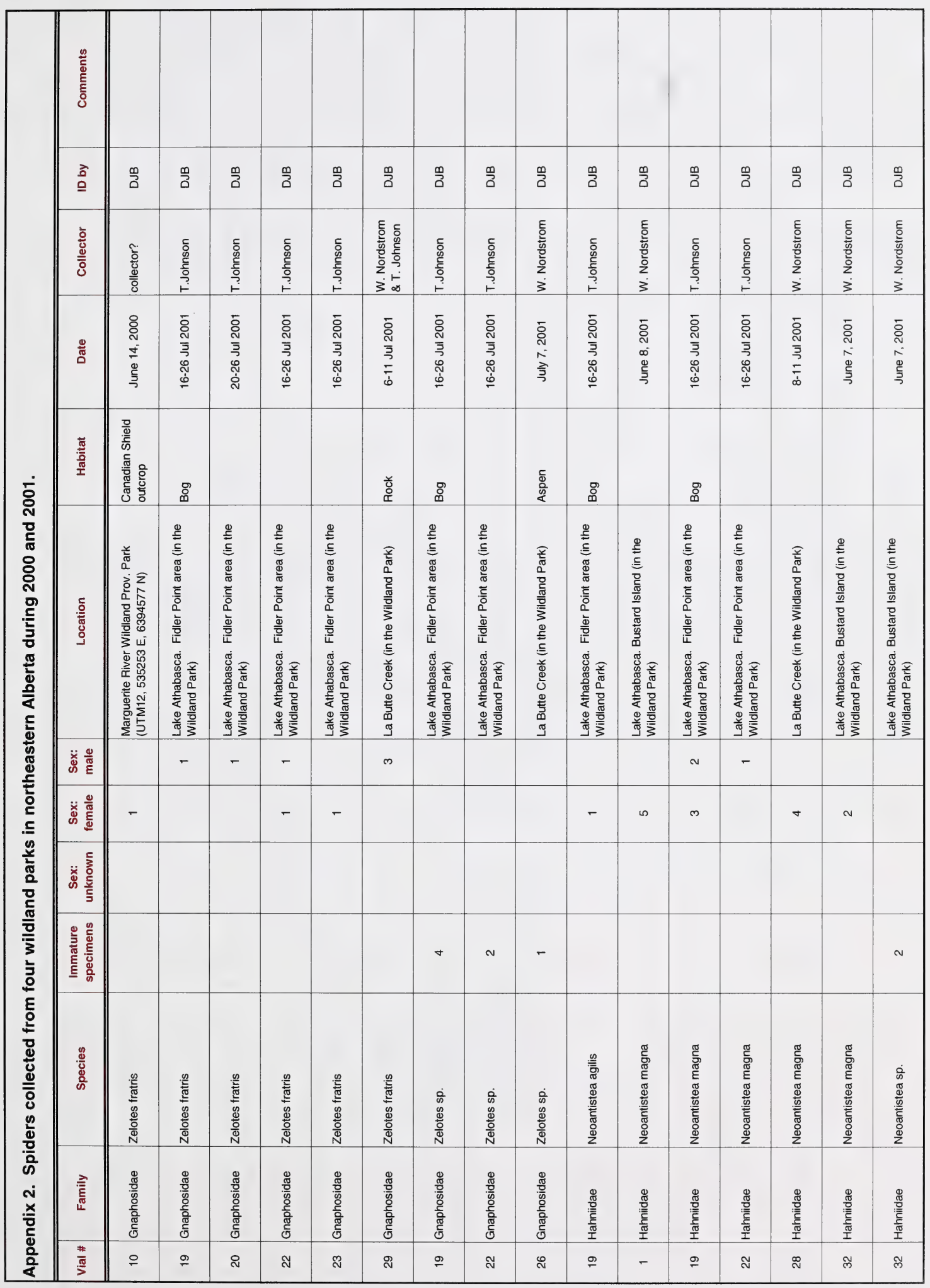





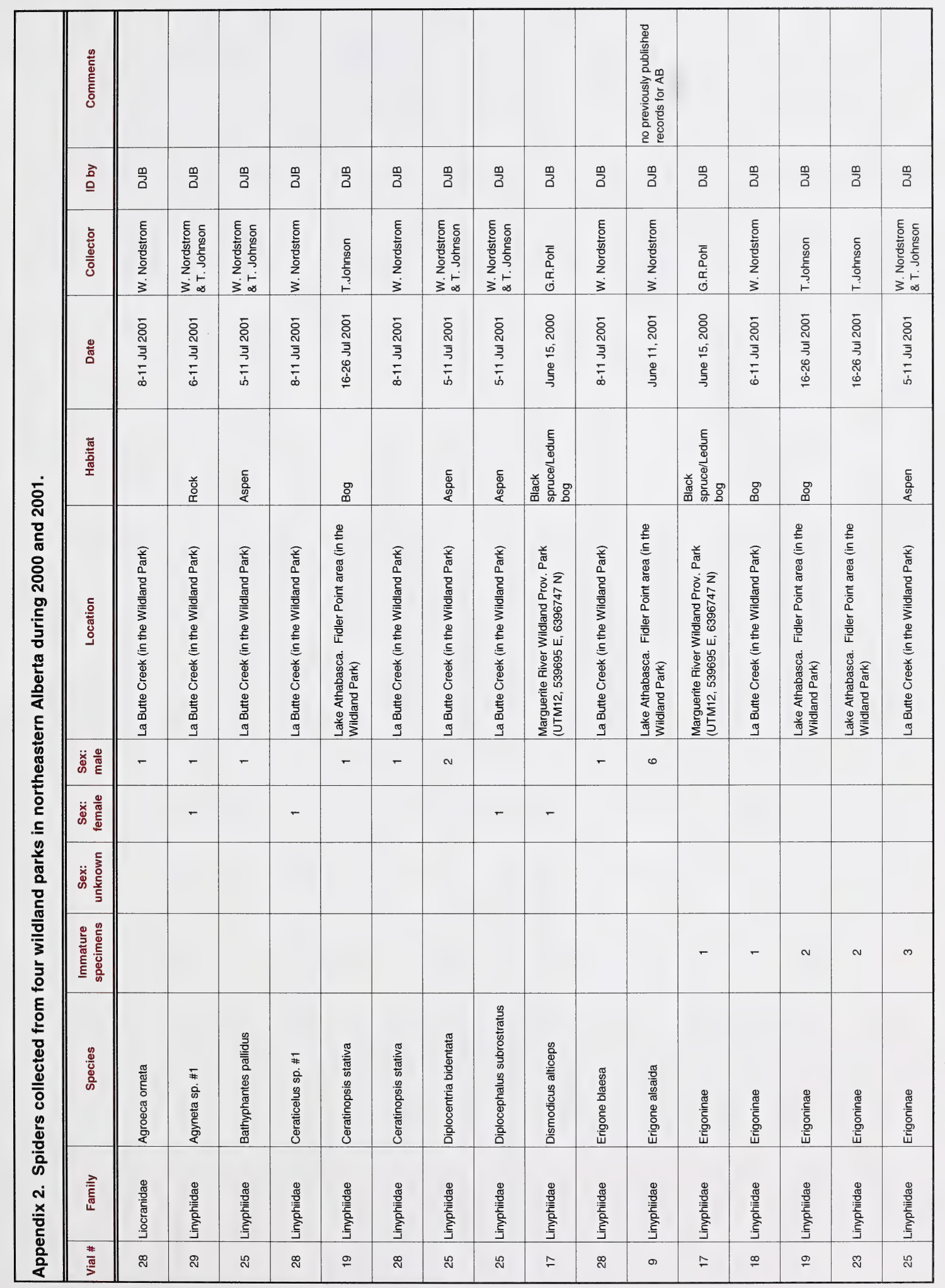





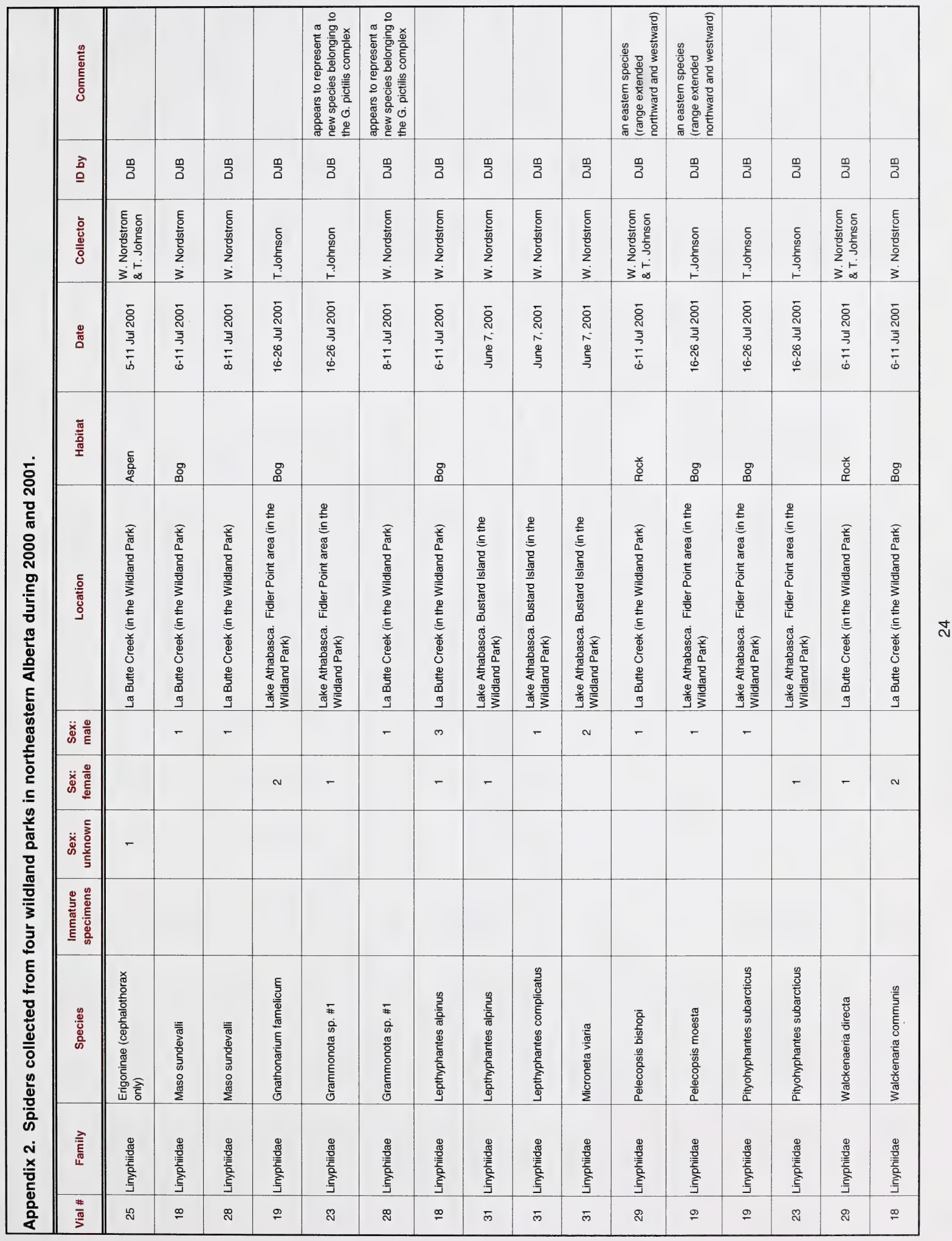




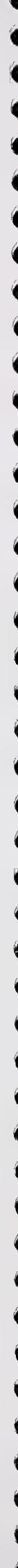




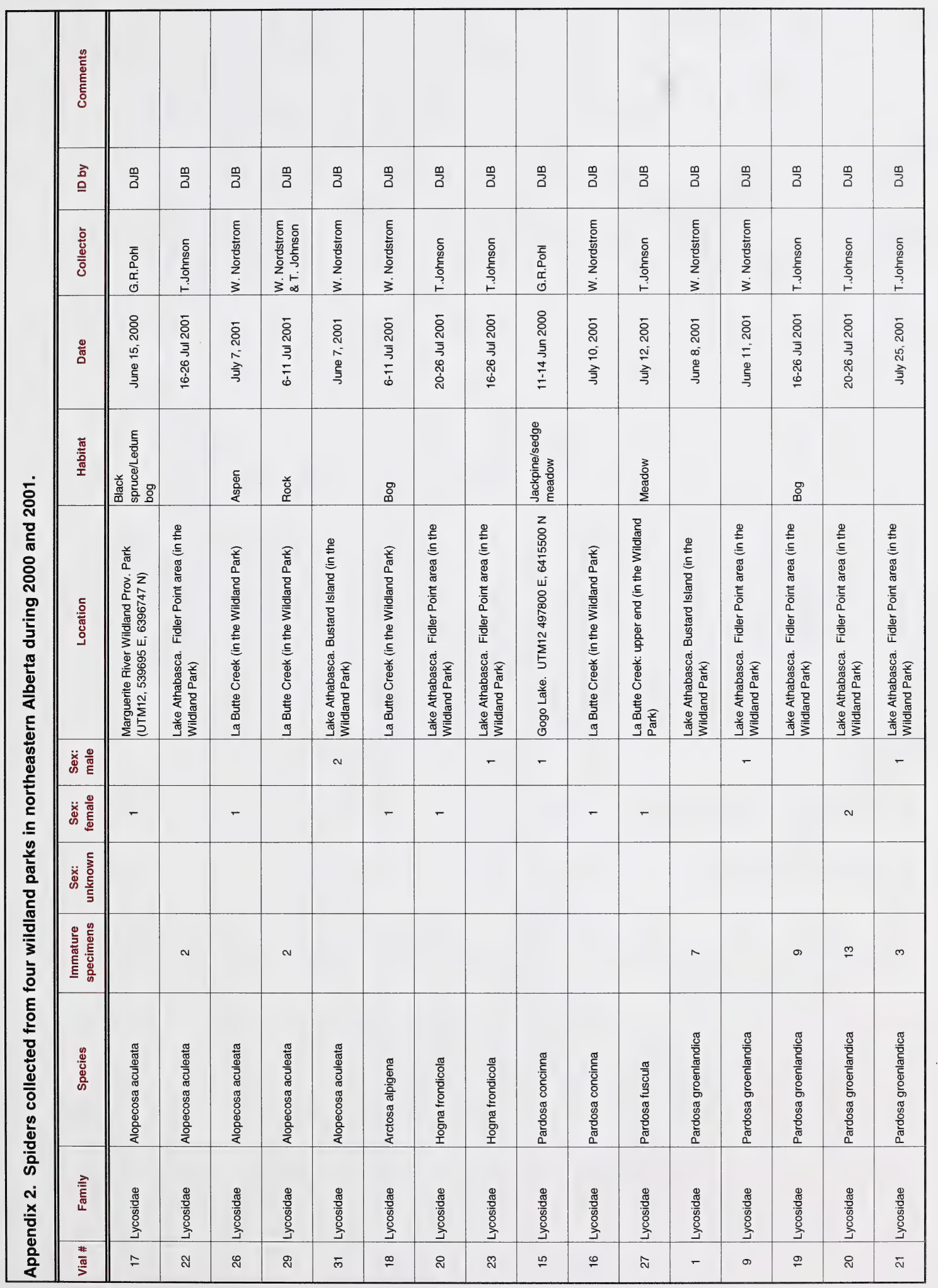





\begin{tabular}{|c|c|c|c|c|c|c|c|c|c|c|c|c|c|c|c|c|c|}
\hline \multirow{12}{*}{ 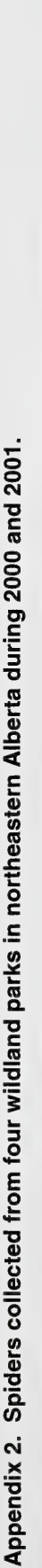 } & $\begin{array}{l}\vec{a} \\
\underline{\varrho}\end{array}$ & $\stackrel{m}{\Delta}$ & $\stackrel{m}{\Delta}$ & $\stackrel{\text { m }}{\Delta}$ & $\stackrel{m}{a}$ & $\stackrel{\text { m }}{\mathrm{a}}$ & $\stackrel{m}{a}$ & 品 & 罟 & $\stackrel{\text { M }}{\Delta}$ & $\stackrel{m}{\Delta}$ & $\stackrel{m}{\Delta}$ & $\stackrel{m}{a}$ & $\stackrel{m}{a}$ & $\stackrel{m}{a}$ & 罟 & $\stackrel{m}{a}$ \\
\hline & $\begin{array}{l}\text { o } \\
\bar{d} \\
\overline{0} \\
\end{array}$ & 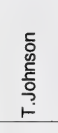 & 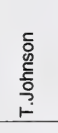 & $\begin{array}{l}\frac{5}{0} \\
\frac{0}{50} \\
\frac{50}{20} \\
2 \\
3\end{array}$ & 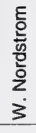 & 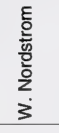 & 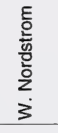 & 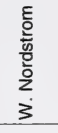 & 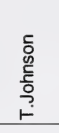 & 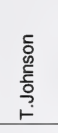 & 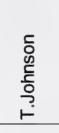 & 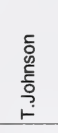 & 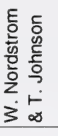 & 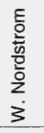 & $\begin{array}{l}\bar{\Sigma} \\
0 \\
0 \\
\frac{0}{\pi} \\
0 \\
0\end{array}$ & 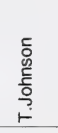 & 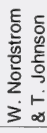 \\
\hline & 总 & 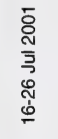 & 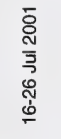 & 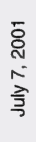 & $\begin{array}{l}\overline{0} \\
\bar{N} \\
\overline{5} \\
\bar{\sigma} \\
\bar{\infty}\end{array}$ & 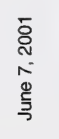 & 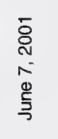 & 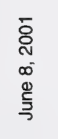 & 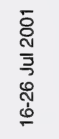 & 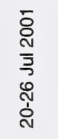 & 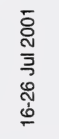 & 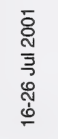 & $\begin{array}{l}\overline{\mathbf{D}} \\
\overline{5} \\
\overline{5} \\
\overline{\overline{0}}\end{array}$ & 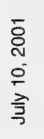 & 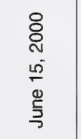 & 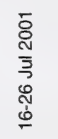 & $\begin{array}{l}\overline{0} \\
\stackrel{0}{n} \\
3 \\
\overline{1} \\
\overline{0}\end{array}$ \\
\hline & 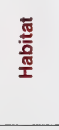 & & & $\begin{array}{l}\frac{5}{0} \\
\frac{0}{8}\end{array}$ & & & & & 足 & & & & $\begin{array}{l}\text { ․ } \\
\text { वृ }\end{array}$ & & 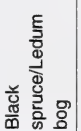 & & $\begin{array}{l}\text { ㅁ } \\
\text { o }\end{array}$ \\
\hline & 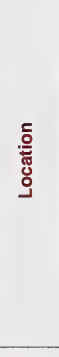 & 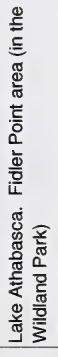 & 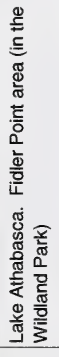 & 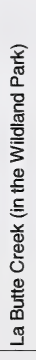 & 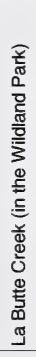 & 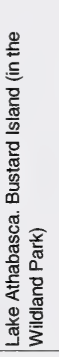 & 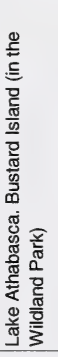 & 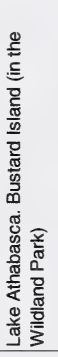 & 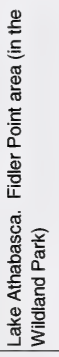 & 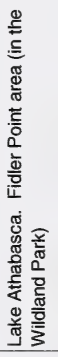 & 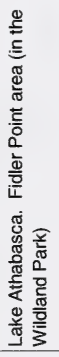 & 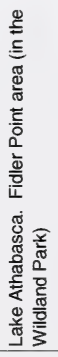 & 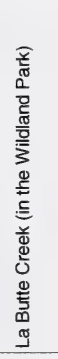 & 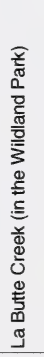 & 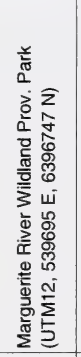 & 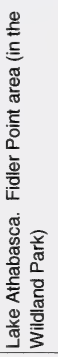 & 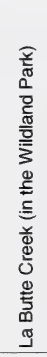 \\
\hline & 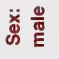 & & $\sim$ & - & & & - & $\sigma$ & & & & & - & & - & - & \\
\hline & 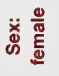 & & & $N$ & $\sim$ & & - & - & - & & & - & & - & & - & - \\
\hline & 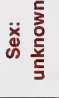 & & & & & & & & & & & & & & & & \\
\hline & 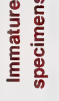 & \pm & \pm & $\stackrel{m}{9}$ & $m$ & - & 0 & $\simeq$ & $\backsim$ & - & - & - & & & & & \\
\hline & $\begin{array}{l}\frac{\mathscr{\Xi}}{\mathrm{d}} \\
\stackrel{\mathrm{g}}{\mathrm{c}}\end{array}$ & 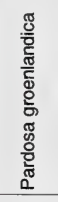 & 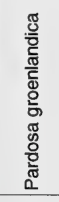 & 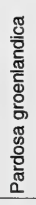 & 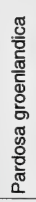 & 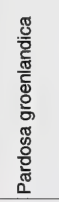 & 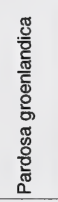 & 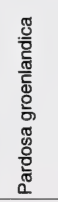 & 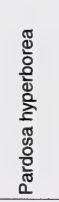 & 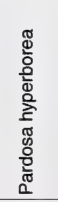 & 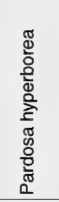 & 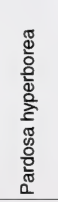 & 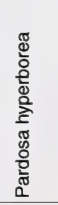 & 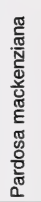 & 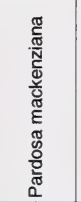 & 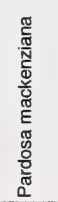 & 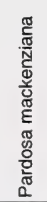 \\
\hline & స్ & $\begin{array}{l}\frac{\mathscr{8}}{0} \\
\frac{0}{0} \\
\frac{0}{3} \\
\frac{3}{3}\end{array}$ & $\begin{array}{l}\frac{0}{0} \\
\frac{\pi}{00} \\
00 \\
0 \\
3 \\
3\end{array}$ & 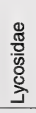 & $\begin{array}{l}\text { 离 } \\
\frac{0}{0} \\
0 \\
0\end{array}$ & 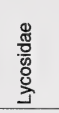 & 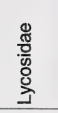 & $\begin{array}{l}\frac{\mathbb{\pi}}{0} \\
\frac{0}{0} \\
\frac{8}{3} \\
3\end{array}$ & 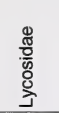 & 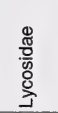 & 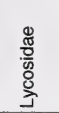 & $\begin{array}{l}\frac{8}{0} \\
\frac{\pi}{00} \\
0 \\
0 \\
3 \\
\end{array}$ & 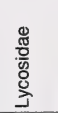 & 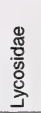 & 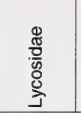 & 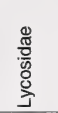 & 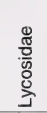 \\
\hline & 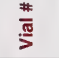 & ส & $\stackrel{\mathbb{N}}{2}$ & $\stackrel{\mathbb{N}}{1}$ & $\stackrel{\infty}{\sim}$ & " & స్ & ల్ల & $\stackrel{-}{2}$ & ฉิ & N & $\cong$ & ని & $\underline{\varphi}$ & $=$ & N & ని \\
\hline
\end{tabular}




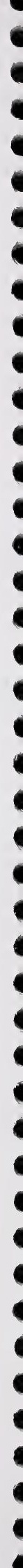




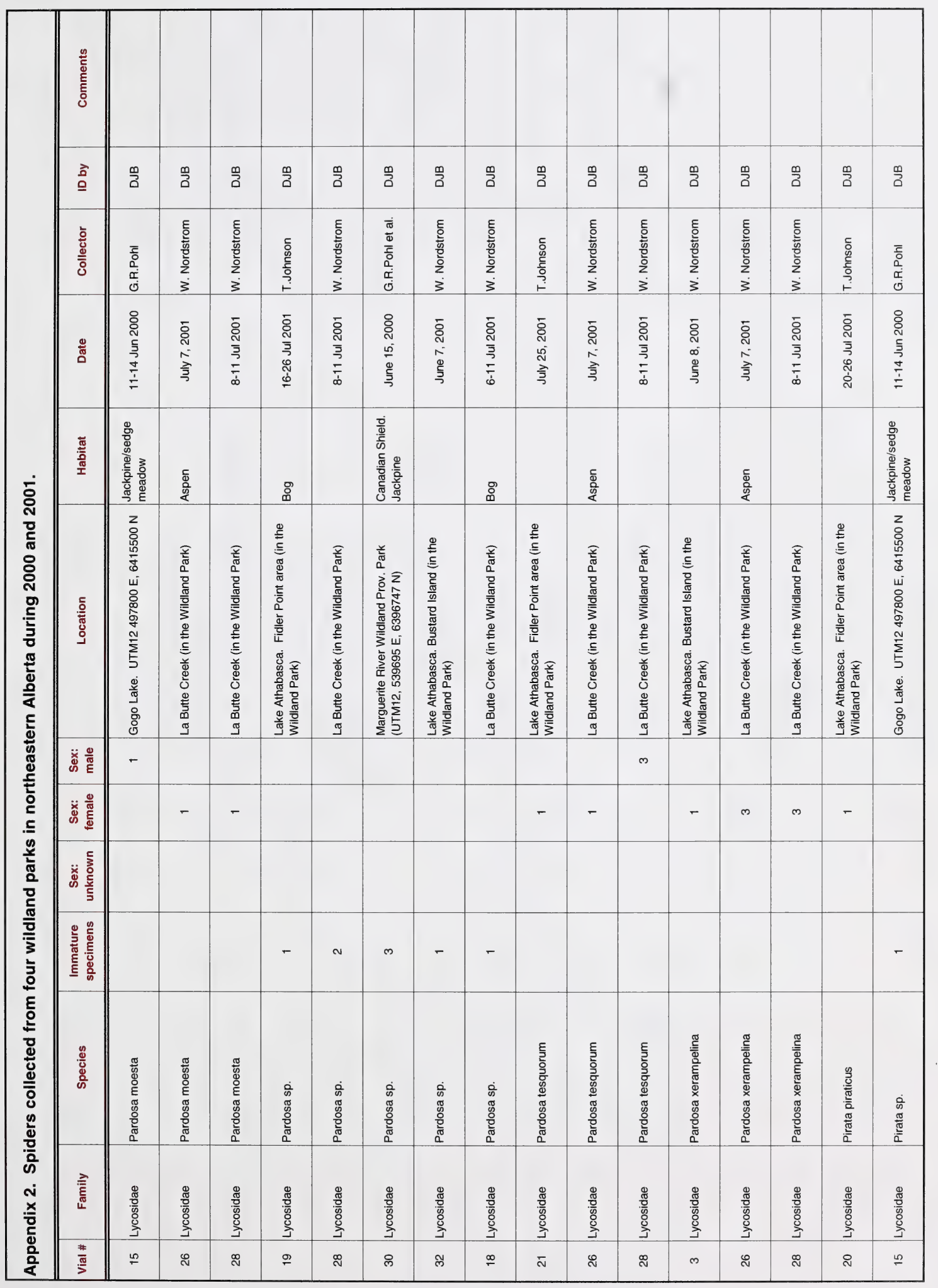





\begin{tabular}{|c|c|c|c|c|c|c|c|c|c|c|c|c|c|c|c|c|c|}
\hline \multirow{13}{*}{ 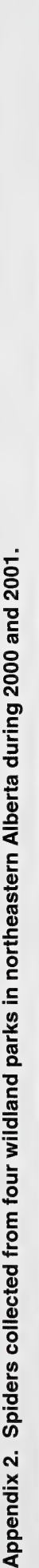 } & 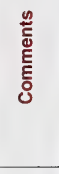 & & 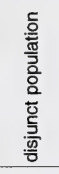 & 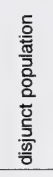 & & & & & & & & & & & & & \\
\hline & $\begin{array}{l}\vec{a} \\
\underline{0}\end{array}$ & $\stackrel{m}{a}$ & $\stackrel{m}{a}$ & $\stackrel{\text { og }}{a}$ & $\stackrel{\mathrm{m}}{\mathrm{a}}$ & $\stackrel{m}{\Delta}$ & $\stackrel{m}{\partial}$ & $\stackrel{m}{a}$ & $\stackrel{m}{a}$ & $\stackrel{m}{a}$ & $\stackrel{m}{a}$ & $\stackrel{M}{0}$ & $\stackrel{\text { m }}{a}$ & $\stackrel{m}{a}$ & $\stackrel{m}{a}$ & $\stackrel{m}{a}$ & 罢 \\
\hline & 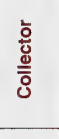 & 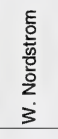 & 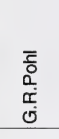 & 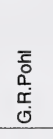 & 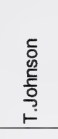 & 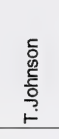 & 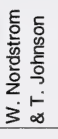 & 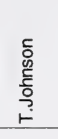 & 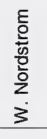 & 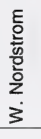 & 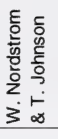 & 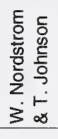 & 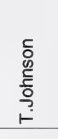 & 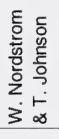 & 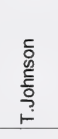 & 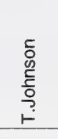 & 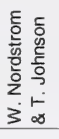 \\
\hline & 造 & $\begin{array}{l}\overline{8} \\
\bar{N} \\
\infty \\
\Phi \\
\Phi \\
5\end{array}$ & 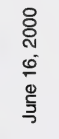 & 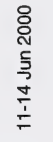 & 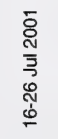 & 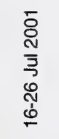 & $\begin{array}{l}\overline{0} \\
\text { N } \\
\overline{5} \\
\bar{b} \\
\bar{b}\end{array}$ & 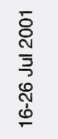 & 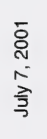 & $\begin{array}{l}\overline{\bar{\delta}} \\
\bar{N} \\
\overline{3} \\
\bar{c} \\
\bar{\infty}\end{array}$ & $\begin{array}{l}\bar{o} \\
\bar{N} \\
\overline{3} \\
\overline{\bar{b}}\end{array}$ & 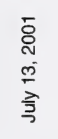 & 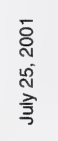 & 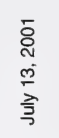 & 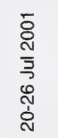 & 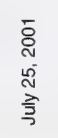 & 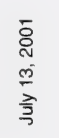 \\
\hline & 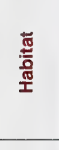 & & & 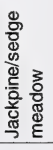 & & & 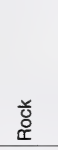 & : & 要 & & $\begin{array}{l}\text { ․ } \\
\text { व. }\end{array}$ & & & & & & \\
\hline & 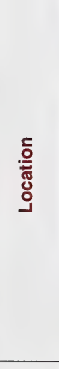 & 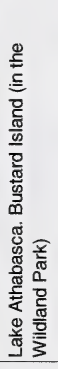 & 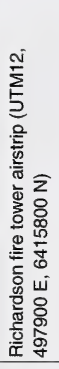 & 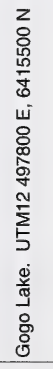 & 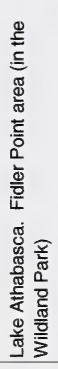 & 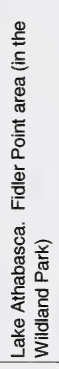 & 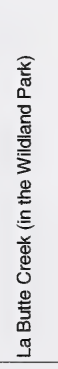 & 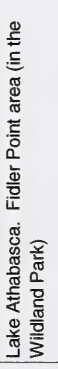 & 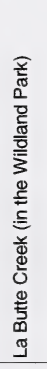 & 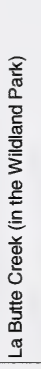 & 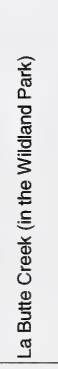 & 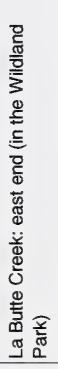 & 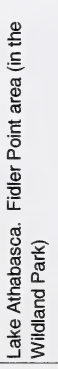 & 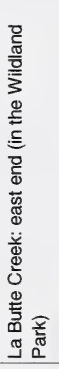 & 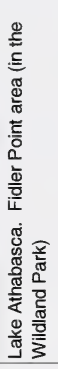 & 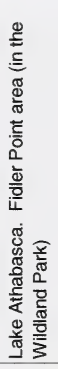 & 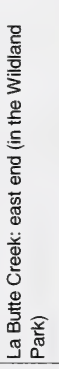 \\
\hline & 苂 & & & - & & & & & & & & & & & & & \\
\hline & 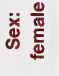 & & - & & - & - & & & & & & & - & - & & & \\
\hline & 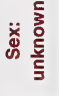 & & & & & & & & & & & - & & & & & \\
\hline & 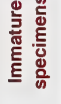 & N & & & - & & - & - & $\infty$ & $\sigma$ & $\infty$ & & & & - & N & - \\
\hline & $\begin{array}{l}\frac{\mathscr{\Xi}}{ \pm} \\
\text { कू }\end{array}$ & 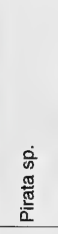 & 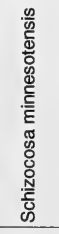 & 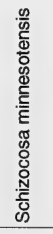 & 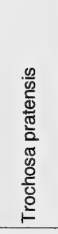 & 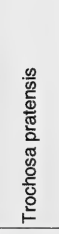 & 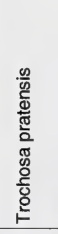 & 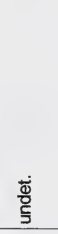 & $\begin{array}{l}\text { 离 } \\
\text { cँ }\end{array}$ & $\begin{array}{l}\frac{\overrightarrow{\mathrm{d}}}{\mathrm{c}} \\
\stackrel{\mathrm{c}}{5}\end{array}$ & $\begin{array}{l}\dot{\mathrm{\Phi}} \\
\mathrm{g}\end{array}$ & 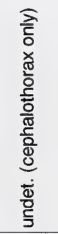 & 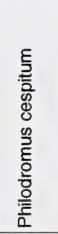 & 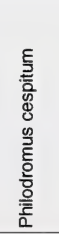 & 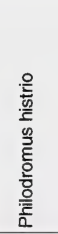 & 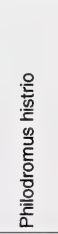 & 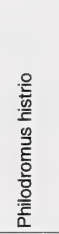 \\
\hline & 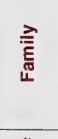 & 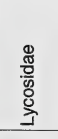 & 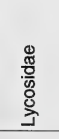 & 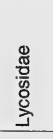 & 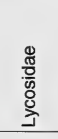 & 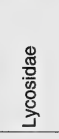 & 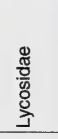 & 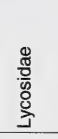 & 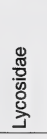 & 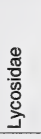 & 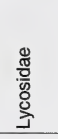 & 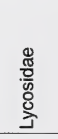 & 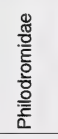 & 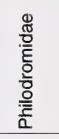 & 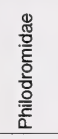 & 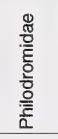 & 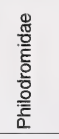 \\
\hline & 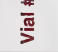 & ల్ల & $\simeq$ & 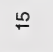 & $\approx$ & 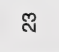 & ฉి & 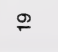 & $\stackrel{\sim}{*}$ & 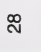 & ని & $\stackrel{\text { d }}{ }$ & $\bar{N}$ & $\stackrel{\mathbb{N}}{\mathrm{N}}$ & $\stackrel{\text { N }}{\text { s. }}$ & $\bar{N}$ & $\stackrel{\sim}{\sim}$ \\
\hline
\end{tabular}




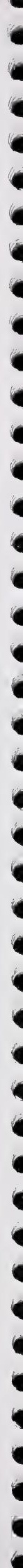




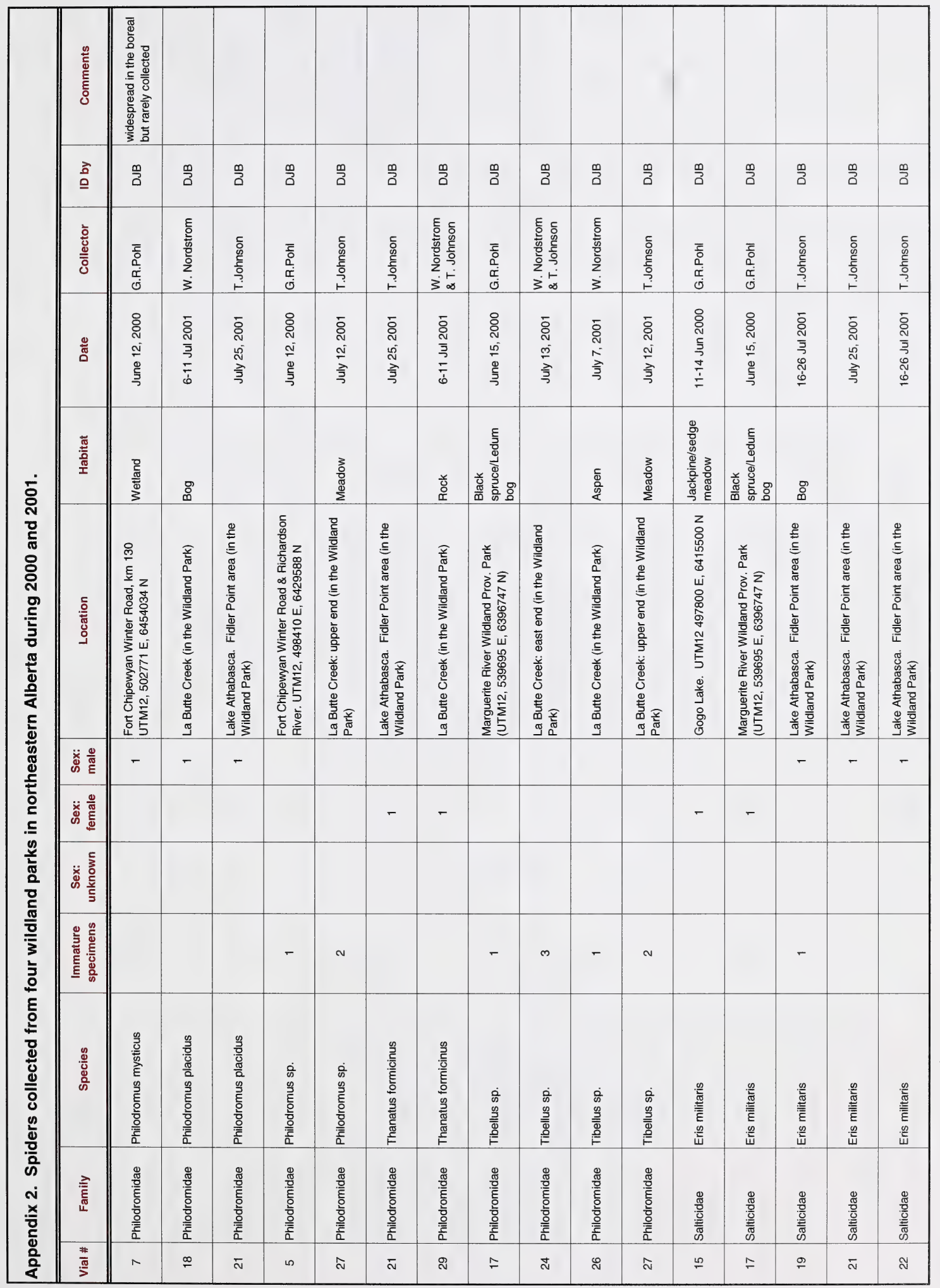





\begin{tabular}{|c|c|c|c|c|c|c|c|c|c|c|c|c|c|c|c|c|c|}
\hline & 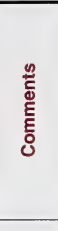 & & & & & 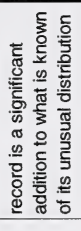 & 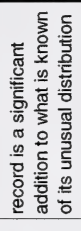 & & & & & & & & & & \\
\hline & $\begin{array}{l}\vec{a} \\
\underline{\underline{a}}\end{array}$ & $\stackrel{m}{0}$ & $\stackrel{m}{0}$ & $\stackrel{m}{\Delta}$ & $\stackrel{m}{\Delta}$ & 咅 & $\stackrel{\text { m }}{\mathrm{a}}$ & $\stackrel{m}{\Delta}$ & $\stackrel{m}{\Delta}$ & $\stackrel{m}{\Delta}$ & $\stackrel{m}{a}$ & $\stackrel{m}{0}$ & $\stackrel{m}{0}$ & $\stackrel{m}{\mathrm{O}}$ & $\stackrel{m}{a}$ & $\stackrel{m}{a}$ & $\stackrel{m}{0}$ \\
\hline & $\begin{array}{l}\stackrel{\bar{o}}{\mathrm{~d}} \\
\overline{\mathrm{\omega}} \\
\overline{\mathrm{o}}\end{array}$ & 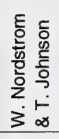 & 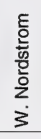 & 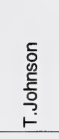 & 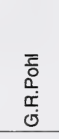 & $\begin{array}{l}\overline{\bar{\delta}} \\
0 \\
\frac{0}{\alpha^{2}} \\
0 \\
0\end{array}$ & 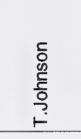 & $\begin{array}{l}\bar{z} \\
0 \\
0 \\
\alpha^{2} \\
0 \\
0\end{array}$ & 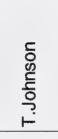 & 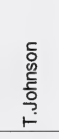 & 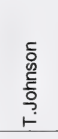 & 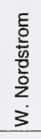 & 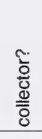 & 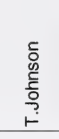 & 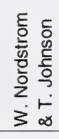 & 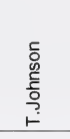 & 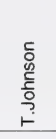 \\
\hline & ฐั๊ & 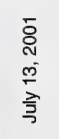 & $\begin{array}{l}\bar{\delta} \\
\text { స̃ } \\
\text { సे }\end{array}$ & 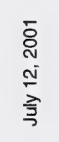 & 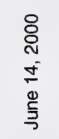 & 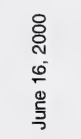 & 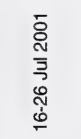 & 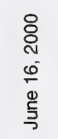 & 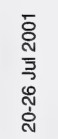 & 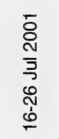 & 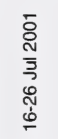 & 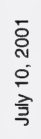 & $\begin{array}{l}\bar{\delta} \\
\text { N } \\
\bar{o} \\
\text { 文 }\end{array}$ & 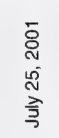 & 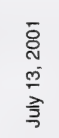 & 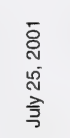 & \begin{tabular}{l}
$\overline{0}$ \\
$\stackrel{N}{ }$ \\
wn \\
\multirow{3}{3}{}
\end{tabular} \\
\hline$\overline{8}$ & 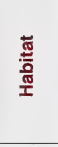 & & 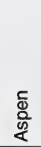 & 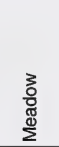 & & & : & & & & & & 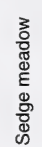 & & & & \\
\hline 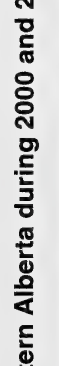 & 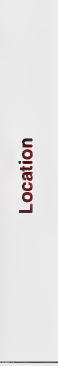 & 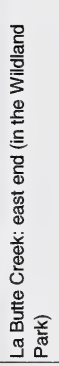 & 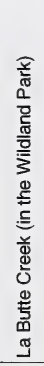 & 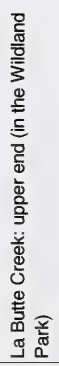 & 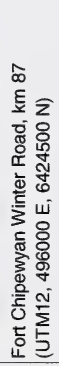 & 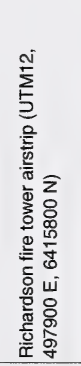 & 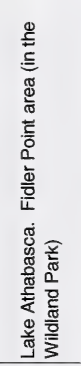 & 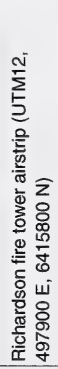 & 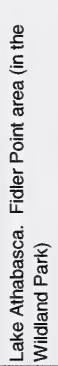 & 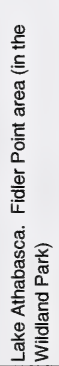 & 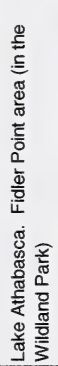 & 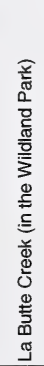 & 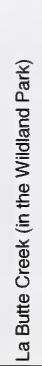 & 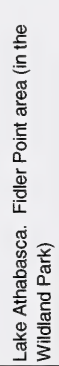 & 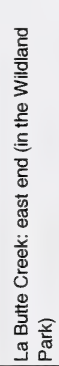 & 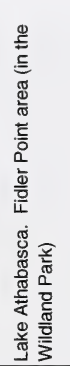 & 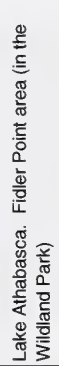 \\
\hline 巴્心 & 苂 弚 & - & - & $\infty$ & & - & - & - & - & - & - & & & & & - & \\
\hline $\begin{array}{l}\stackrel{2}{0} \\
. \subseteq\end{array}$ & 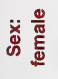 & & & - & & & & & & & & & & & - & & is \\
\hline 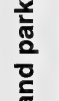 & 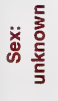 & & & & & & & & & & & & & & & & \\
\hline $\begin{array}{l}\frac{0}{3} \\
3 \\
0 \\
0\end{array}$ & 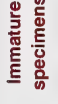 & & & 0 & - & & & & & & & $\sim$ & - & - & & & \\
\hline 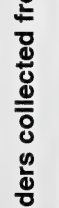 & $\begin{array}{l}\frac{\mathscr{E}}{\tilde{\Phi}} \\
\text { कू }\end{array}$ & 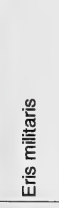 & 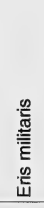 & 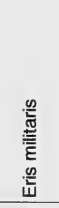 & $\begin{array}{l}\frac{1}{0} \\
\frac{0}{4} \\
\frac{0}{4}\end{array}$ & 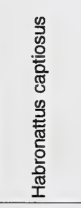 & 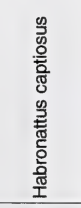 & 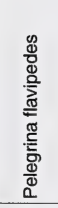 & 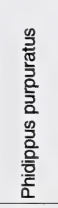 & 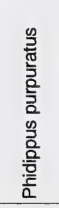 & 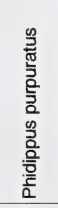 & 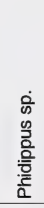 & $\begin{array}{l}\dot{\overrightarrow{\mathrm{d}}} \\
\mathrm{g}\end{array}$ & $\begin{array}{l}\frac{\dot{\mathrm{s}}}{\mathrm{c}} \\
\mathrm{z}\end{array}$ & 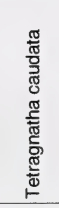 & 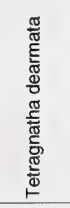 & 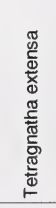 \\
\hline $\begin{array}{l}\text { ஸे } \\
\text { ஸे } \\
\grave{x} \\
\ddot{0}\end{array}$ & 恴 & 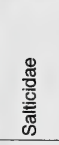 & 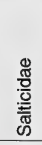 & 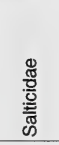 & 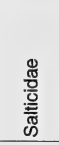 & 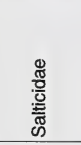 & 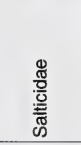 & 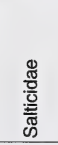 & 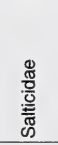 & 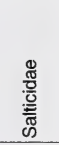 & 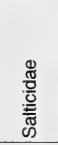 & 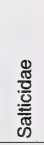 & 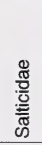 & 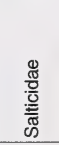 & 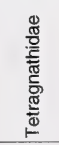 & 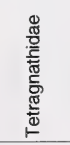 & 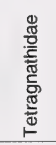 \\
\hline$\frac{0}{\frac{0}{2}}$ & 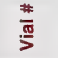 & N & 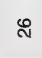 & $\hat{N}$ & $\sigma$ & $\cong$ & $\stackrel{2}{ }$ & $\simeq$ & i & $\approx$ & 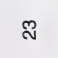 & $\stackrel{\varphi}{0}$ & $=$ & $\bar{N}$ & \& & $\bar{N}$ & $\bar{N}$ \\
\hline
\end{tabular}




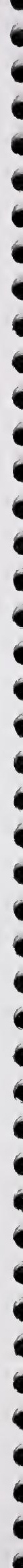




\begin{tabular}{|c|c|c|c|c|c|c|c|c|c|c|c|c|c|c|c|c|c|}
\hline & $\stackrel{\vec{a}}{\underline{a}}$ & $\stackrel{m}{a}$ & $\stackrel{m}{a}$ & $\stackrel{m}{a}$ & $\stackrel{m}{a}$ & $\stackrel{m}{a}$ & $\stackrel{m}{a}$ & 眾 & $\stackrel{m}{a}$ & 孚 & $\stackrel{m}{\circ}$ & $\stackrel{m}{a}$ & $\stackrel{\text { ğ }}{a}$ & $\stackrel{\text { qu }}{a}$ & $\stackrel{m}{a}$ & 鲾 & 睘 \\
\hline & $\begin{array}{l}\stackrel{\bar{o}}{\mathrm{~d}} \\
\overline{\mathrm{e}} \\
\overline{\mathrm{u}}\end{array}$ & 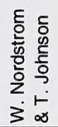 & 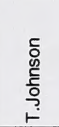 & 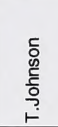 & 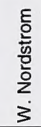 & 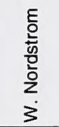 & 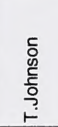 & 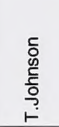 & 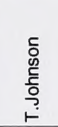 & 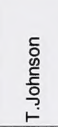 & 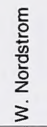 & 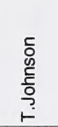 & $\begin{array}{l}\dot{\sigma} \\
\bar{\sigma} \\
\frac{\sigma}{\sigma} \\
0 \\
\dot{\sigma} \\
\dot{0}\end{array}$ & 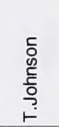 & $\begin{array}{l}\bar{z} \\
0 \\
0 \\
\text { d. } \\
0\end{array}$ & 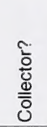 & 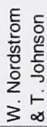 \\
\hline & ๕ัँ & $\begin{array}{l}\overline{8} \\
\stackrel{N}{ } \\
m \\
\bar{m} \\
\overline{3}\end{array}$ & 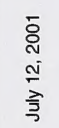 & 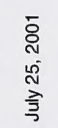 & 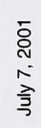 & 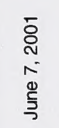 & 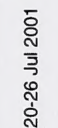 & 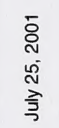 & 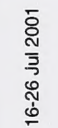 & 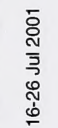 & 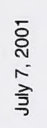 & 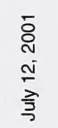 & 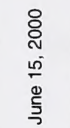 & 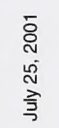 & 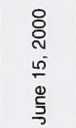 & 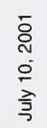 & 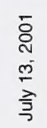 \\
\hline$\dot{8}$ & 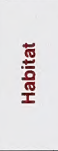 & & 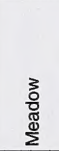 & & $\begin{array}{l}\frac{c}{\Phi} \\
\frac{8}{8}\end{array}$ & & & & & & $\begin{array}{l}\frac{5}{\Phi} \\
\frac{0}{8} \\
\frac{2}{8}\end{array}$ & 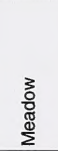 & 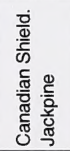 & & 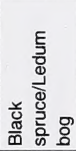 & & \\
\hline 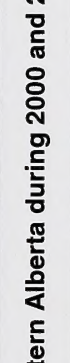 & 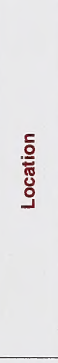 & 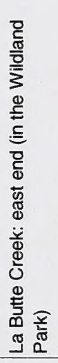 & 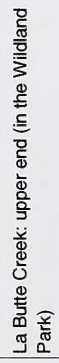 & 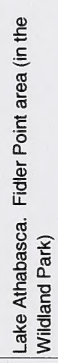 & 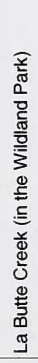 & 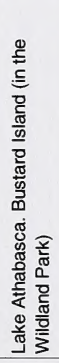 & 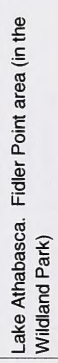 & 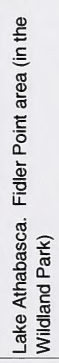 & 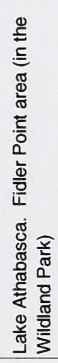 & 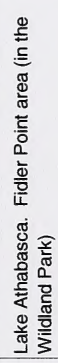 & 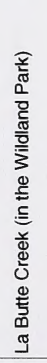 & 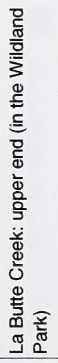 & 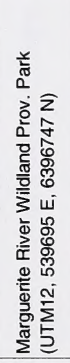 & 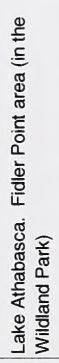 & 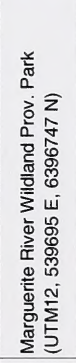 & 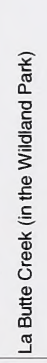 & 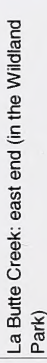 \\
\hline छू & 苂 & - & & - & & & & $\sim$ & & & - & & $\sim$ & & & & \\
\hline $\begin{array}{l}\text { to } \\
. \leq\end{array}$ & 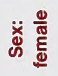 & $\sim$ & $\sim$ & & & & N & $\infty$ & - & $\sim$ & - & $\sim$ & & - & & & I \\
\hline 를 & 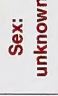 & & & & & & & & & & & & & & & & \\
\hline $\begin{array}{l}\frac{0}{3} \\
3 \\
5 \\
0\end{array}$ & 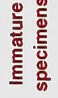 & & & & $\sim$ & - & & & & & & & $m$ & & - & - & $\sim$ \\
\hline 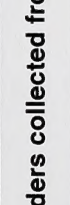 & 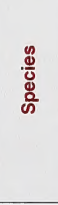 & 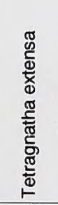 & 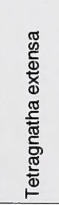 & 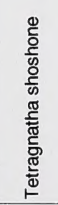 & 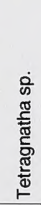 & 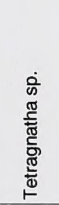 & 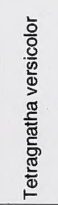 & 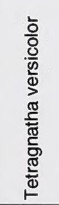 & 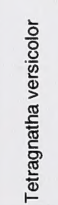 & 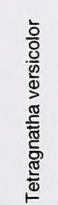 & 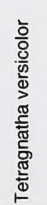 & 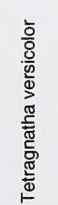 & 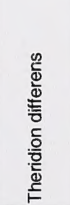 & 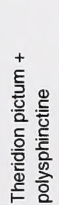 & 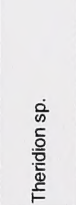 & 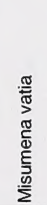 & 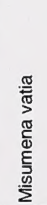 \\
\hline $\begin{array}{l}\text { क̊ } \\
\text { ஸ் } \\
\grave{x}\end{array}$ & 츹 & 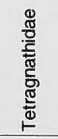 & 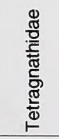 & 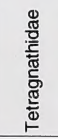 & 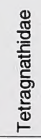 & 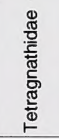 & 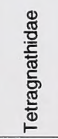 & 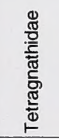 & 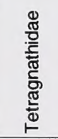 & 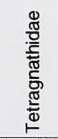 & 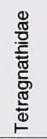 & 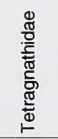 & 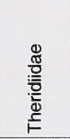 & 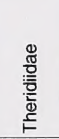 & 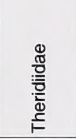 & 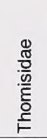 & 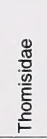 \\
\hline$\frac{2}{2}$ & 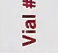 & A & $\hat{N}$ & $\bar{N}$ & $\stackrel{8}{\circ}$ & ల్ల & ని & $\bar{N}$ & $\approx$ & $\approx$ & $\stackrel{\sim}{\sim}$ & กิ & \& & $\bar{N}$ & $=$ & 0 & d \\
\hline
\end{tabular}





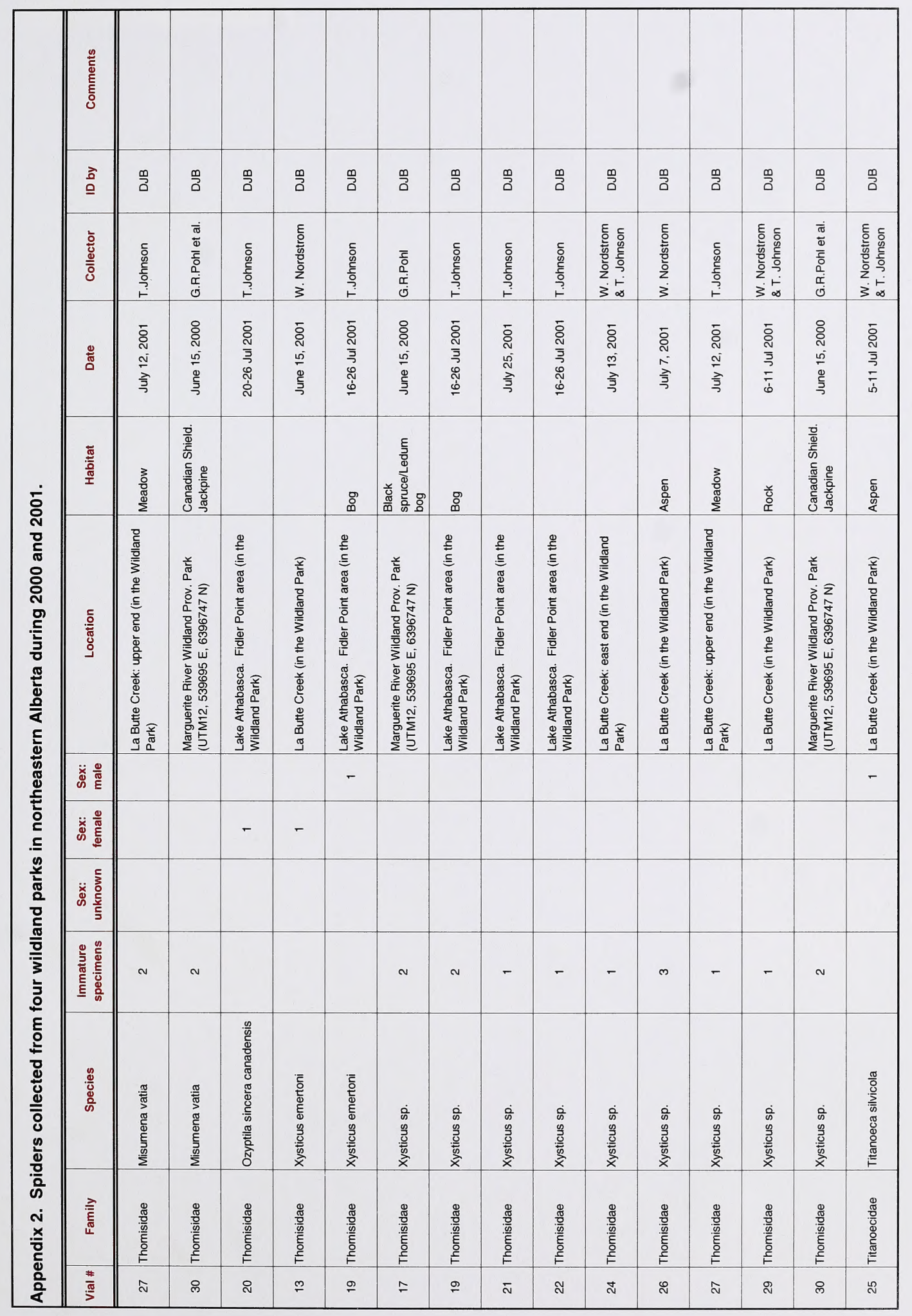


NATIONAL LIBRARY OF CANADA
Bibliothèque nationale du Canada

33286530274550 